\title{
Topology optimization of a flexible multibody system with variable-length bodies described by ALE-ANCF
}

Sun, Jialiang; Tian, Qiang; Hu, Haiyan; Pedersen, Niels L.

Published in:

Nonlinear Dynamics

Link to article, DOI:

10.1007/s11071-018-4201-6

Publication date:

2018

Document Version

Peer reviewed version

Link back to DTU Orbit

Citation (APA):

Sun, J., Tian, Q., Hu, H., \& Pedersen, N. L. (2018). Topology optimization of a flexible multibody system with variable-length bodies described by ALE-ANCF. Nonlinear Dynamics, 93(2), 413-441.

https://doi.org/10.1007/s11071-018-4201-6

\section{General rights}

Copyright and moral rights for the publications made accessible in the public portal are retained by the authors and/or other copyright owners and it is a condition of accessing publications that users recognise and abide by the legal requirements associated with these rights.

- Users may download and print one copy of any publication from the public portal for the purpose of private study or research.

- You may not further distribute the material or use it for any profit-making activity or commercial gain

- You may freely distribute the URL identifying the publication in the public portal

If you believe that this document breaches copyright please contact us providing details, and we will remove access to the work immediately and investigate your claim 


\title{
Topology optimization of a flexible multibody system
} with variable-length bodies described by ALE-ANCF

\author{
Jialiang Sun ${ }^{\mathrm{a}}$, Qiang Tian ${ }^{\mathrm{b}}$, Haiyan $\mathrm{Hu}^{\mathrm{a}, \mathrm{b}, *}$, Niels L. Pedersen ${ }^{\mathrm{c}}$
}

${ }^{\text {a }}$ State Key Laboratory of Mechanics and Control of Mechanical Structures, College of Aerospace Engineering, Nanjing University of Aeronautics and Astronautics, Nanjing 210016, China

E-mail address: sunjialiang@nuaa.edu.cn;

*hhyae@nuaa.edu.cn

${ }^{\mathrm{b}}$ MOE Key Laboratory of Dynamics and Control of Flight Vehicle,

School of Aerospace Engineering, Beijing Institute of Technology, Beijing 100081, China E-mail address: tianqiang_hust@aliyun.com

${ }^{\mathrm{c}}$ Department of Mechanical Engineering,

Solid Mechanics, Technical University of Denmark, Kgs. Lyngby 2800, Denmark

E-mail address: nlp@mek.dtu.dk

* Author to whom all correspondence should be addressed 


\begin{abstract}
Recent years have witnessed the application of topology optimization to flexible multibody systems (FMBS) so as to enhance their dynamic performances. In this study, an explicit topology optimization approach is proposed for an FMBS with variablelength bodies via the moving morphable components (MMC). Using the arbitrary Lagrangian-Eulerian (ALE) formulation, the thin plate elements of the absolute nodal coordinate formulation (ANCF) are used to describe the plate-like bodies with variablelength. For the thin plate element of ALE-ANCF, the elastic force and additional inertial force, as well as their Jacobians, are analytically deduced. In order to account for the variable design domain, the sets of equivalent static loads (ESL) are reanalyzed by introducing the actual and virtual design domains so as to transform the topology optimization problem of dynamic response into a static one. Finally, the novel MMCbased topology optimization method is employed to solve the corresponding static topology optimization problem by explicitly evolving the shapes and orientations of a set of structural components. The effect of the minimum feature size on the optimization of an FMBS is studied. Three numerical examples are presented to validate the accuracy of the thin plate element of ALE-ANCF and the efficiency of the proposed topology optimization approach, respectively.
\end{abstract}

Keywords Flexible multibody dynamics • Arbitrary Lagrangian-Eulerian formulation - Absolute nodal coordinate formulation - Topology optimization • Moving morphable components 


\section{Introduction}

Flexible multibody systems (FMBS) with variable-length bodies serve as useful models for a wide range of industrial products, such as cranes, elevators, pipes conveying fluids, cable-driven parallel manipulators, tethered satellites, telescoping boom systems and deployable solar sails [1-8]. Fig. 1 shows the first stage deployment of a spinning solar sail, similar to the problem studied by Sakamoto et al. [6]. During the deployment, four flexible beams extend from a spinning rigid hub and their lengths are variable. Previous studies in this field focused on the dynamic modeling and analysis of one-dimensional systems, like wire ropes, cables and belts [1-5, 9-11]. For a planar or spatial FMBS with variable-length bodies, such as a solar sail [6] or a deployable articulated mast $[8,12]$, neither dynamic modeling nor optimal design has received much attention.

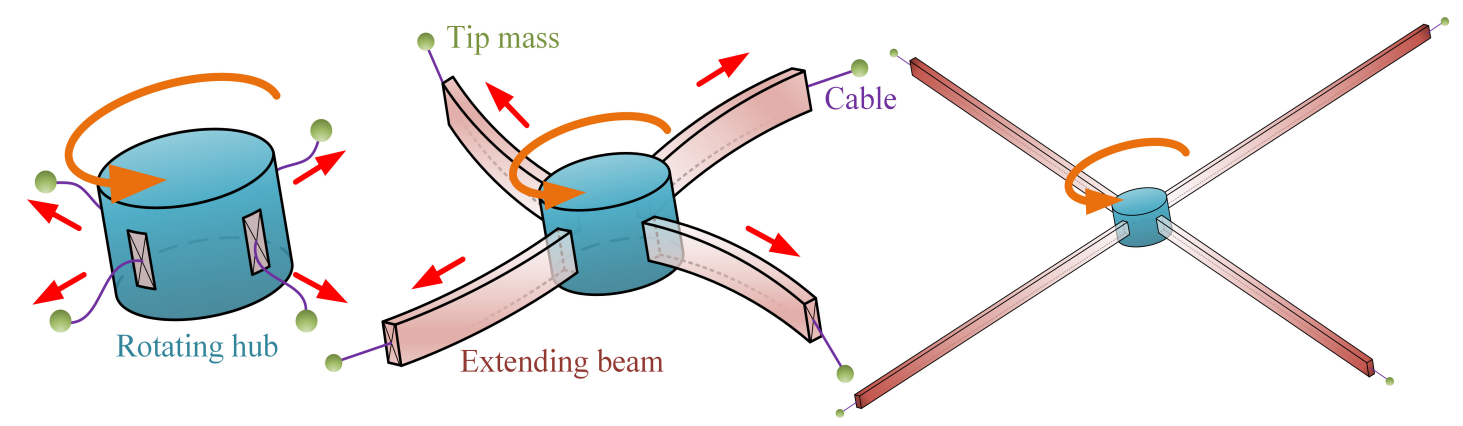

Fig. 1 The first stage deployment of a spinning solar sail [6]

Topology optimization of an FMBS has called considerable attention in recent years to reduce the moving weight, improve the energy efficiency and enhance the dynamic performance. Since the pioneering work by Bendsøe and Kikuchi [13], several approaches [14] have been studied for the topology optimization of fluids, acoustics, electromagnetics and optics, as well as FMBS [15-19], but not yet for any FMBS with variable-length subject to both large overall motion and large deformation. This is probably due to the fact that the variable-length gives rise to three challenges for the topology optimization.

The first challenge is how to accurately describe the FMBS. At present, many formulations can be used to describe the FMBS, such as the floating frame of reference formulation (FFRF), the absolute nodal coordinate formulation (ANCF) [20] and the large rotation vector formulation (LRVF) [21]. Ding et al. [22] has clearly illustrated the performance of the LRVF. For the simulation of an FMBS with variable-length 
bodies, however, only two formulations are available. One is the variable-domain finite element (VFE) model based on the FFRF [23, 24]. The other is the arbitrary Lagrangian-Eulerian (ALE) description based on the ANCF [25]. Nevertheless, the VFE-FFRF cannot account for the large deformations of an FMBS with variable-length bodies due to the inherent nature of FFRF [15]. In the ALE-ANCF, additional material coordinates, besides the node positions and their slopes, are introduced as generalized coordinates $[2,3,25]$. Consequently, different from the conventional nodes tied to material points in ANCF, the nodes in ALE-ANCF are not associated with specific material points. When the material coordinates in ALE-ANCF are fixed, the finite element of ALE-ANCF becomes the conventional finite element of ANCF. The material coordinates of the ALE-ANCF give rise to a variable shape function matrix, and as a consequence, a variable mass matrix and additional inertial forces. Many kinds of finite elements of ALE-ANCF have been proposed for different applications [2-4, $11,26]$, including a thin plate element of ALE-ANCF proposed first by Hyldahl et al. [26]. Their studies, however, have not given the analytical formulae of the elastic force and additional inertial force, as well as the corresponding Jacobians.

The second challenge is how to deal with the dynamic characteristics concerned with the variable design domain. In the conventional topology optimization of an FMBS, the design domain is prescribed and constant. Consequently, the topology optimization can be performed using the method of the equivalent static loads (ESL) or directly from the dynamic simulation of an FMBS. The former is named the ESL method $[15,18,27-$ 31 ], while the latter is called the integrated optimization method [16, 32]. For an FMBS with variable-length bodies, the design domain varies over time. It is therefore hard to perform the topology optimization of an FMBS with variable-length bodies using the integrated optimization method. By introducing the concepts of actual and virtual design domains, the ESL sets are redefined. Taking the extension case of a flexible body as an example, the stretching domain is called the actual design domain, while the unstretched domain is the so-called virtual design domain. The whole design domain, which is the union of the actual and virtual design domains, is time-invariant. The ESL sets are separately analyzed for the actual and virtual design domains. In the actual design domain, the ESL sets are computed by multiplying the nonlinear stiffness matrix and the deformations of the flexible bodies [15], while in the virtual design domain, the ESL sets are identically equal to zeros. Thus, the dynamic topology optimization of an FMBS with variable-length bodies can be turned into a static one under multiple 
equivalent static loading conditions.

The third challenge is how to describe the structural topology and how to efficiently solve the corresponding topology optimization problem. Previous studies on the topology optimization of an FMBS mainly employ the density method and the level set method (LSM). Held et al. [17] studied the effect of the model reduction of FFRF on the topology optimization results of an FMBS by using the solid isotropic material with penalization (SIMP) approach. Moghadasi et al. [18] involved an accurate revolute joints and bearing domains model into the topology optimization of an FMBS via SIMP approach. Tromme et al. [16] employed an LSM to optimize the flexible bodies of an FMBS and used elliptical holes to generate new topologies. Sun et al. [15] utilized a semi-implicit LSM to perform the topology optimization of an FMBS described by ANCF. However, the traditional pixel-based density method and node point-based LSM describe the structural topology and perform the topology optimization implicitly. As a result, the two methods can have a large number of design variables, and might also have difficulty in precisely controlling the feature sizes of a component. It is noteworthy that recently, the moving morphable components (MMC) based topology optimization method has been first extended to the optimization of a spatial FMBS undergoing both large overall motions and large deformations [19]. The MMC-based topology optimization method [33-37] has a potential to overcome the shortcomings of the traditional density method and LSM as mentioned above by performing topology optimization explicitly and geometrically. According to the MMC method, a set of structural components serve as the building blocks, and enable one to get the optimal topology by optimizing the positions and shapes of the components. As a result, the MMC method can not only directly link with computer-aided design (CAD) modeling, but also easily control the feature sizes, like the minimum thickness of a component.

This paper aims at solving the above problems in the following sections. Section 2 introduces the fundamentals of the thin plate element of ALE-ANCF, presents the efficient schemes of the elastic forces, additional inertial forces and the corresponding Jacobian formulations, and gives the dynamic equation of an FMBS with variablelength bodies. Section 3 outlines the MMC-based topology optimization method, and presents the ESL-based computation flow of the topology optimization for an FMBS with variable-length bodies. Section 4 includes three numerical examples to validate the accuracy of the thin plate element of ALE-ANCF and the efficiency of the topology optimization approach. Finally, Section 5 gives the concluding remarks. 


\section{Flexible multibody dynamics with variable-length bodies}

The rectangular thin plate element of ALE-ANCF was first proposed by Hyldahl et al. [26] by combining the ALE framework and ANCF and ignoring the transverse shear deformation. However, they did not derive the efficient computational schemes for the elastic force vector, additional inertial force vector and their Jacobians. This section, hence, presents these schemes for the later use in topology optimization.

2.1 Implementation of a thin plate element of ALE-ANCF

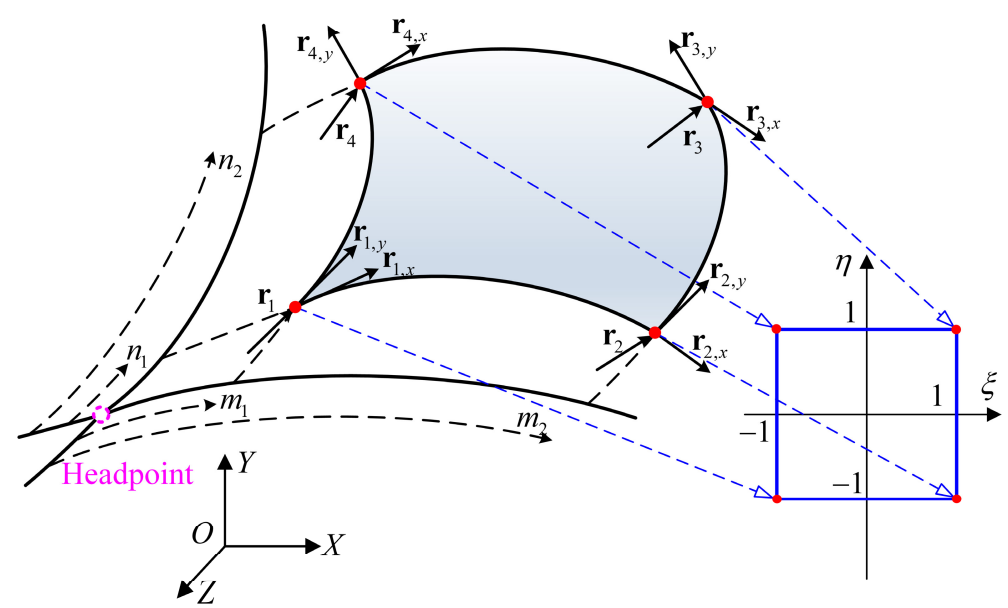

Fig. 2 A deformed thin plate element of ALE-ANCF

Different from the rectangular thin plate element of ANCF [38], the rectangular thin plate element of ALE-ANCF in Fig. 2 uses the global position vectors, in-plane slope vectors, as well as the local material coordinates as the generalized coordinates, that is,

$$
\mathbf{q}=\left[\begin{array}{ll}
\mathbf{q}_{e}^{\mathrm{T}} & \mathbf{q}_{l}^{\mathrm{T}}
\end{array}\right]^{\mathrm{T}}
$$

where $\quad \mathbf{q}_{e}=\left[\begin{array}{lllllll}\mathbf{r}_{1}^{\mathrm{T}} & \mathbf{r}_{1, x}^{\mathrm{T}} & \mathbf{r}_{1, y}^{\mathrm{T}} & \cdots & \mathbf{r}_{4}^{\mathrm{T}} & \mathbf{r}_{4, x}^{\mathrm{T}} & \mathbf{r}_{4, y}^{\mathrm{T}}\end{array}\right]^{\mathrm{T}}$ is the vector of ANCF coordinates and $\mathbf{q}_{l}=\left[\begin{array}{llll}m_{1} & n_{1} & m_{2} & n_{2}\end{array}\right]^{\mathrm{T}}$ is the vector of local material coordinates.

The position vector of an arbitrary point in the rectangular thin plate element can be described as

$$
\mathbf{r}=\mathbf{N}_{e} \mathbf{q}_{e}
$$

where $\mathbf{N}_{e}=\left[\begin{array}{lllll}N_{1} \cdot \mathbf{I}_{3 \times 3} & N_{2} \cdot \mathbf{I}_{3 \times 3} & N_{3} \cdot \mathbf{I}_{3 \times 3} & \cdots & N_{12} \cdot \mathbf{I}_{3 \times 3}\end{array}\right]$ is the matrix of $C^{1}$ 
continuous interpolation functions with entries determined as follows

$$
\begin{aligned}
& N_{1}=\frac{1}{16}(\xi-1)^{2}(2+\xi)(\eta-1)^{2}(2+\eta), N_{2}=\frac{a}{32}(\xi-1)^{2}(\xi+1)(\eta-1)^{2}(2+\eta), \\
& N_{3}=\frac{b}{32}(\xi-1)^{2}(2+\xi)(\eta-1)^{2}(\eta+1), N_{4}=\frac{1}{16}(\xi+1)^{2}(2-\xi)(\eta-1)^{2}(2+\eta), \\
& N_{5}=\frac{a}{32}(\xi+1)^{2}(\xi-1)(\eta-1)^{2}(2+\eta), N_{6}=\frac{b}{32}(\xi+1)^{2}(2-\xi)(\eta-1)^{2}(\eta+1), \\
& N_{7}=\frac{1}{16}(\xi+1)^{2}(2-\xi)(\eta+1)^{2}(2-\eta), N_{8}=\frac{a}{32}(\xi+1)^{2}(\xi-1)(\eta+1)^{2}(2-\eta), \\
& N_{9}=\frac{b}{32}(\xi+1)^{2}(2-\xi)(\eta+1)^{2}(\eta-1), N_{10}=\frac{1}{16}(\xi-1)^{2}(2+\xi)(\eta+1)^{2}(2-\eta), \\
& N_{11}=\frac{a}{32}(\xi-1)^{2}(\xi+1)(\eta+1)^{2}(2-\eta), N_{12}=\frac{b}{32}(\xi-1)^{2}(2+\xi)(\eta+1)^{2}(\eta-1) .
\end{aligned}
$$

In Eq. (3), $\quad \xi=\frac{2 m-m_{1}-m_{2}}{m_{2}-m_{1}} \quad\left(m_{1} \leq m \leq m_{2}\right) \quad$ and $\eta=\frac{2 n-n_{1}-n_{2}}{n_{2}-n_{1}} \quad\left(n_{1} \leq n \leq n_{2}\right)$ are the isoparametric coordinates, and $a=m_{2}-m_{1}$ and $b=n_{2}-n_{1}$ are the length and the width of the undeformed thin plate element, respectively.

According to Eq. (2), the velocity and acceleration vectors of an arbitrary point can be expressed as

$$
\dot{\mathbf{r}}=\mathbf{N} \cdot \dot{\mathbf{q}}, \ddot{\mathbf{r}}=\mathbf{N} \cdot \ddot{\mathbf{q}}+\ddot{\mathbf{r}}_{p},
$$

where $\mathbf{N}=\left[\begin{array}{llllllll}\mathbf{N}_{e} & \mathbf{N}_{e, m_{1}} \mathbf{q}_{e} & \mathbf{N}_{e, n_{1}} \mathbf{q}_{e} & \mathbf{N}_{e, m_{2}} \mathbf{q}_{e} & \mathbf{N}_{e, n_{2}} \mathbf{q}_{e}\end{array}\right]$ is the shape function matrix. $\ddot{\mathbf{r}}_{p}$ is the term that results in the additional inertial force, and can be expressed as

$$
\begin{aligned}
\ddot{\mathbf{r}}_{p} & =\left(2 \dot{m}_{1} \mathbf{N}_{e, m_{1}}+2 \dot{n}_{1} \mathbf{N}_{e, n_{1}}+2 \dot{m}_{2} \mathbf{N}_{e, m_{2}}+2 \dot{n}_{2} \mathbf{N}_{e, n_{2}}\right) \dot{\mathbf{q}}_{e} \\
& +\left(\dot{m}_{1}^{2} \mathbf{N}_{e, m_{1} m_{1}}+\dot{n}_{1}^{2} \mathbf{N}_{e, n_{1} n_{1}}+\dot{m}_{2}^{2} \mathbf{N}_{e, m_{2} m_{2}}+\dot{n}_{2}^{2} \mathbf{N}_{e, n_{2} n_{2}}\right) \mathbf{q}_{e} \\
& +\left(2 \dot{m}_{1} \dot{n}_{1} \mathbf{N}_{e, m_{1} n_{1}}+2 \dot{m}_{1} \dot{m}_{2} \mathbf{N}_{e, m_{1} m_{2}}+2 \dot{m}_{1} \dot{n}_{2} \mathbf{N}_{e, m_{1} n_{2}}\right) \mathbf{q}_{e} \\
& +\left(2 \dot{n}_{1} \dot{m}_{2} \mathbf{N}_{e, n_{1} m_{2}}+2 \dot{n}_{1} \dot{n}_{2} \mathbf{N}_{e, n_{1} n_{2}}+2 \dot{m}_{2} \dot{n}_{2} \mathbf{N}_{e, m_{2} n_{2}}\right) \mathbf{q}_{e}
\end{aligned}
$$

Here, $\mathbf{N}_{e, m_{1}}$ denotes $\frac{\partial \mathbf{N}_{e}}{\partial m_{1}}$, and $\mathbf{N}_{e, m_{1} m_{1}}$ denotes $\frac{\partial^{2} \mathbf{N}_{e}}{\partial m_{1} \partial m_{1}}$.

\subsection{Formulations of the elastic force and its Jacobian}

The strain energy of the thin plate element can be divided into two terms, that is, the in-plane term $U^{\varepsilon}$ due to the longitudinal and shear deformations in the mid-plane and the out-of-plane term $U^{\kappa}$ due to the bending and twist deformations as follows $[26,38,39]$ 


$$
U=U^{\varepsilon}+U^{\kappa}=\frac{1}{2} \int_{V_{0}} \boldsymbol{\varepsilon}^{\mathrm{T}} \mathbf{E}^{\varepsilon} \boldsymbol{\varepsilon} \mathrm{d} V+\frac{1}{2} \int_{V_{0}} \boldsymbol{\kappa}^{\mathrm{T}} \mathbf{E}^{\kappa} \boldsymbol{\kappa} \mathrm{d} V,
$$

where $V_{0}$ is the initial volume of the thin plate element, $\boldsymbol{\varepsilon}$ and $\boldsymbol{\kappa}$ are the mid-plane strain tensor and curvature vector as follows

$$
\boldsymbol{\varepsilon}=\left[\begin{array}{lll}
\frac{\mathbf{r}_{, m}^{\mathrm{T}} \mathbf{r}_{, m}-1}{2} & \frac{\mathbf{r}_{, n}^{\mathrm{T}} \mathbf{r}_{, n}-1}{2} & \mathbf{r}_{, m}^{\mathrm{T}} \mathbf{r}_{, n}
\end{array}\right]^{\mathrm{T}}, \mathbf{\kappa}=\left[\begin{array}{lll}
\frac{\mathbf{r}_{, m m}^{\mathrm{T}} \mathbf{n}}{\|\mathbf{n}\|^{3}} & \frac{\mathbf{r}_{, n n}^{\mathrm{T}} \mathbf{n}}{\|\mathbf{n}\|^{3}} & \frac{2 \mathbf{r}_{, m n}^{\mathrm{T}} \mathbf{n}}{\|\mathbf{n}\|^{3}}
\end{array}\right]^{\mathrm{T}},
$$

with $\mathbf{n}=\mathbf{r}_{, m} \times \mathbf{r}_{, n}$. In Eq. (6), $\mathbf{E}^{\varepsilon}$ and $\mathbf{E}^{\kappa}$ are the elastic coefficient matrix under the assumption of plane stress, and are given by

$$
\mathbf{E}^{\varepsilon}=\frac{E}{1-v^{2}}\left[\begin{array}{ccc}
1 & v & 0 \\
v & 1 & 0 \\
0 & 0 & (1-v) / 2
\end{array}\right], \mathbf{E}^{\kappa}=\frac{c^{2}}{12} \mathbf{E}^{\varepsilon},
$$

where $E$ denotes Young's modulus, $v$ denotes Poisson's ratio and $c$ is the thickness of the thin plate.

According to the work of Sanborn et al. [40], the interpolation function matrix in Eq. (3) and the strain energy in Eq. (6) of the thin plate element may result in the curve-induced distortion and membrane locking. However, the membrane locking occurs only during the bending of the thin plate element [40]. The present study mainly focuses on the topology optimization of the variable-length bodies in an FMBS undergoing only planar overall motions. Therefore, these variable-length bodies undergo the in-plane longitudinal and shear deformations, rather than the out-of-plane bending and twist deformations. The study, hence, does not account for the membrane locking problem of the thin plate element of ALE-ANCF. In addition, Sanborn et al. [40] also proposed the flat-mapped extension modeling method to address the curveinduced distortion and membrane locking in 1-axis bending problems of the thin plate element of ANCF. It is possible, thus, to introduce the method into further studies to achieve accurate results. More specific information can be found in [40].

According to Eq. (6), the elastic force vector is defined as

$$
\mathbf{F}^{e}=\mathbf{F}^{\varepsilon}+\mathbf{F}^{\kappa}=\left(\frac{\partial U^{\varepsilon}}{\partial \mathbf{q}}\right)^{\mathrm{T}}+\left(\frac{\partial U^{\kappa}}{\partial \mathbf{q}}\right)^{\mathrm{T}}
$$

where $\mathbf{F}^{\varepsilon}$ is the elastic force vector associated with in-plane deformations and $\mathbf{F}^{\kappa}$ is the elastic force vector associated with out-of-plane deformations. For the planar topology optimization problems of stiffness design, the out-of-plane strain energy $U^{\kappa}$ can be omitted as it contributes little to the elastic force [15]. To reduce the 
computational cost associated with the in-plane elastic force vector $\mathbf{F}^{\varepsilon}$ and the corresponding Jacobian formulation, efficient schemes are derived in this study by using the invariant matrix method [41].

The in-plane elastic force vector $\mathbf{F}^{\varepsilon}$ in Eq. (9) can be expressed as

$$
\mathbf{F}^{\varepsilon}=\left(\frac{\partial U^{\varepsilon}}{\partial \mathbf{q}}\right)^{\mathrm{T}}=\int_{V_{0}}\left(\frac{\partial \boldsymbol{\varepsilon}}{\partial \mathbf{q}}\right)^{\mathrm{T}} \mathbf{E}^{\varepsilon} \boldsymbol{\varepsilon} \mathrm{d} V .
$$

The entries of the elastic force vector $\mathbf{F}^{\varepsilon}$ can then be efficiently computed as

$$
F_{k}^{\varepsilon}=\left\{\begin{array}{r}
\frac{1}{2}\left[\left(\mathbf{K}_{1}\right)_{i k a b}+\left(\mathbf{K}_{2}\right)_{i k a b}+\left(\mathbf{K}_{3}\right)_{i k a b}\right] q_{i} q_{a} q_{b}-\frac{1}{2}\left[\left(\mathbf{G}_{1}\right)_{i k}+\left(\mathbf{G}_{2}\right)_{i k}\right] q_{i} \\
(k=1, \ldots, 36) \\
\frac{1}{4}\left[\left(\mathbf{K}_{4}\right)_{i j k a b}+\left(\mathbf{K}_{5}\right)_{i j k a b}+\left(\mathbf{K}_{6}\right)_{i j k a b}\right] q_{i} q_{j} q_{a} q_{b}-\frac{1}{4}\left[\left(\mathbf{G}_{3}\right)_{i j k}+\left(\mathbf{G}_{4}\right)_{i j k}\right] q_{i} q_{j} \\
(k=37, \ldots, 40)
\end{array} .\right.
$$

In Eq. (11), the subscripts $i, j, a, b=1,2, \ldots, 36$ and the detailed expressions of $\left(\mathbf{K}_{1}\right)_{i k a b}, \quad\left(\mathbf{K}_{2}\right)_{i k a b}, \quad\left(\mathbf{K}_{3}\right)_{i k a b}, \quad\left(\mathbf{K}_{4}\right)_{i j k a b}, \quad\left(\mathbf{K}_{5}\right)_{i j k a b}, \quad\left(\mathbf{K}_{6}\right)_{i j k a b},\left(\mathbf{G}_{1}\right)_{i k},\left(\mathbf{G}_{2}\right)_{i k}$, $\left(\mathbf{G}_{3}\right)_{i j k}$ and $\left(\mathbf{G}_{4}\right)_{i j k}$ are derived and listed in the Appendix.

According to Eq. (11), the entries of the Jacobian formulation of the elastic force are derived as follows:

when $k=1,2, \ldots, 36$ and $l=1,2, \ldots, 36$,

$$
\begin{aligned}
\frac{\partial F_{k}^{\varepsilon}}{\partial q_{l}} & =\frac{1}{2}\left[\left(\mathbf{K}_{1}\right)_{l k a b}+2\left(\mathbf{K}_{1}\right)_{a k l b}+\left(\mathbf{K}_{2}\right)_{l k a b}+2\left(\mathbf{K}_{2}\right)_{a k l b}\right] q_{a} q_{b} \\
& +\frac{1}{2}\left[\left(\mathbf{K}_{3}\right)_{l k a b}+\left(\mathbf{K}_{3}\right)_{a k l b}+\left(\mathbf{K}_{3}\right)_{a k l b}\right] q_{a} q_{b}-\frac{1}{2}\left[\left(\mathbf{G}_{1}\right)_{l k}+\left(\mathbf{G}_{2}\right)_{l k}\right],
\end{aligned}
$$

when $k=1,2, \ldots, 36$ and $l=37,38, \ldots, 40$,

$$
\begin{aligned}
\frac{\partial F_{k}^{\varepsilon}}{\partial q_{l}} & =\frac{1}{2}\left[\left(\mathbf{K}_{1}\right)_{i k a b, q_{l}}+\left(\mathbf{K}_{2}\right)_{i k a b, q_{l}}+\left(\mathbf{K}_{3}\right)_{i k a b, q_{l}}\right] q_{i} q_{a} q_{b} \\
& -\frac{1}{2}\left[\left(\mathbf{G}_{1}\right)_{i k, q_{l}}+\left(\mathbf{G}_{2}\right)_{i k, q_{l}}\right] q_{i},
\end{aligned}
$$

when $k=37,38, \ldots, 40$ and $l=1,2, \ldots, 36$, 


$$
\begin{aligned}
\frac{\partial F_{k}^{\varepsilon}}{\partial q_{l}} & =\frac{1}{4}\left[2\left(\mathbf{K}_{4}\right)_{i l k a b}+2\left(\mathbf{K}_{4}\right)_{i a k l b}+2\left(\mathbf{K}_{5}\right)_{i l k a b}+2\left(\mathbf{K}_{5}\right)_{i a k l b}\right] q_{i} q_{a} q_{b} \\
& +\frac{1}{4}\left[\left(\mathbf{K}_{6}\right)_{l i k a b}+\left(\mathbf{K}_{6}\right)_{i k k a b}+\left(\mathbf{K}_{6}\right)_{i a k l b}+\left(\mathbf{K}_{6}\right)_{i a k b l}\right] q_{i} q_{a} q_{b} \\
& -\frac{1}{4}\left[2\left(\mathbf{G}_{3}\right)_{i l k}+2\left(\mathbf{G}_{4}\right)_{i l k}\right] q_{i},
\end{aligned}
$$

when $k=37,38, \ldots, 40$ and $l=37,38, \ldots, 40$,

$$
\begin{aligned}
\frac{\partial F_{k}^{\varepsilon}}{\partial q_{l}} & =\frac{1}{4}\left[\left(\mathbf{K}_{4}\right)_{i j k a b, q_{l}}+\left(\mathbf{K}_{5}\right)_{i j k a b, q_{l}}+\left(\mathbf{K}_{6}\right)_{i j k a b, q_{l}}\right] q_{i} q_{j} q_{a} q_{b} \\
& -\frac{1}{4}\left[\left(\mathbf{G}_{3}\right)_{i j k, q_{l}}+\left(\mathbf{G}_{4}\right)_{i j k, q_{l}}\right] q_{i} q_{j} .
\end{aligned}
$$

In Eqs. (13) and (15), $\left(\mathbf{K}_{1}\right)_{i k a b, q_{l}}$ represents the partial derivatives of $\left(\mathbf{K}_{1}\right)_{i k a b}$ with respect to the local material coordinates $q_{l}(l=37,38, \ldots, 40)$. The detailed expressions of $\left(\mathbf{K}_{1}\right)_{i k a b, q_{l}}, \quad\left(\mathbf{K}_{2}\right)_{i k a b, q_{l}}, \quad\left(\mathbf{K}_{3}\right)_{i k a b, q_{l}}, \quad\left(\mathbf{K}_{4}\right)_{i j k a b, q_{l}}, \quad\left(\mathbf{K}_{5}\right)_{i j k a b, q_{l}}$, $\left(\mathbf{K}_{6}\right)_{i j k a b, q_{l}},\left(\mathbf{G}_{1}\right)_{i k, q_{l}},\left(\mathbf{G}_{2}\right)_{i k, q_{l}},\left(\mathbf{G}_{3}\right)_{i j k, q_{l}}$ and $\left(\mathbf{G}_{4}\right)_{i j k, q_{l}}$ are derived and listed in the Appendix.

2.3 Formulations of the additional inertial force and its Jacobian

According to Eq. (5), the additional inertial force vector $\mathbf{F}^{a}$ is defined as

$$
\mathbf{F}^{a}=\int_{V_{0}} \rho \mathbf{N}^{\mathrm{T}} \ddot{\mathbf{r}_{p}} \mathrm{~d} V
$$

where $\rho$ is the density. The entries of the additional inertial force vector $\mathbf{F}^{a}$ can be efficiently computed as follows:

when $k=1,2, \ldots, 36$,

$$
\begin{aligned}
F_{k}^{a} & =\left[\dot{m}_{1}\left(\mathbf{H}_{1}\right)_{k a}+\dot{n}_{1}\left(\mathbf{H}_{2}\right)_{k a}+\dot{m}_{2}\left(\mathbf{H}_{3}\right)_{k a}+\dot{n}_{2}\left(\mathbf{H}_{4}\right)_{k a}\right] \dot{q}_{a} \\
& +\left[\dot{m}_{1}^{2}\left(\mathbf{H}_{5}\right)_{k a}+\dot{n}_{1}^{2}\left(\mathbf{H}_{6}\right)_{k a}+\dot{m}_{2}^{2}\left(\mathbf{H}_{7}\right)_{k a}+\dot{n}_{2}^{2}\left(\mathbf{H}_{8}\right)_{k a}\right] q_{a} \\
& +\left[\dot{m}_{1} \dot{n}_{1}\left(\mathbf{H}_{9}\right)_{k a}+\dot{m}_{1} \dot{m}_{2}\left(\mathbf{H}_{10}\right)_{k a}+\dot{m}_{1} \dot{n}_{2}\left(\mathbf{H}_{11}\right)_{k a}\right] q_{a} \\
& +\left[\dot{n}_{1} \dot{m}_{2}\left(\mathbf{H}_{12}\right)_{k a}+\dot{n}_{1} \dot{n}_{2}\left(\mathbf{H}_{13}\right)_{k a}+\dot{m}_{2} \dot{n}_{2}\left(\mathbf{H}_{14}\right)_{k a}\right] q_{a},
\end{aligned}
$$

when $k=37,38, \ldots, 40$, 


$$
\begin{aligned}
F_{k}^{a} & =\left[\dot{m}_{1}\left(\mathbf{H}_{15}\right)_{k b a}+\dot{n}_{1}\left(\mathbf{H}_{16}\right)_{k b a}+\dot{m}_{2}\left(\mathbf{H}_{17}\right)_{k b a}+\dot{n}_{2}\left(\mathbf{H}_{18}\right)_{k b a}\right] q_{b} \dot{q}_{a} \\
& +\left[\dot{m}_{1}^{2}\left(\mathbf{H}_{19}\right)_{k b a}+\dot{n}_{1}^{2}\left(\mathbf{H}_{20}\right)_{k b a}+\dot{m}_{2}^{2}\left(\mathbf{H}_{21}\right)_{k b a}+\dot{n}_{2}^{2}\left(\mathbf{H}_{22}\right)_{k b a}\right] q_{b} q_{a} \\
& +\left[\dot{m}_{1} \dot{n}_{1}\left(\mathbf{H}_{23}\right)_{k b a}+\dot{m}_{1} \dot{m}_{2}\left(\mathbf{H}_{24}\right)_{k b a}+\dot{m}_{1} \dot{n}_{2}\left(\mathbf{H}_{25}\right)_{k b a}\right] q_{b} q_{a} \\
& +\left[\dot{n}_{1} \dot{m}_{2}\left(\mathbf{H}_{26}\right)_{k b a}+\dot{n}_{1} \dot{n}_{2}\left(\mathbf{H}_{27}\right)_{k b a}+\dot{m}_{2} \dot{n}_{2}\left(\mathbf{H}_{28}\right)_{k b a}\right] q_{b} q_{a} .
\end{aligned}
$$

In Eqs. (17) and (18), the subscripts $a, b=1,2, \ldots, 36$ and the detailed expressions of $\left(\mathbf{H}_{1}\right)_{k a},\left(\mathbf{H}_{2}\right)_{k a}, \ldots,\left(\mathbf{H}_{14}\right)_{k a}$ and $\left(\mathbf{H}_{15}\right)_{k b a},\left(\mathbf{H}_{16}\right)_{k b a}, \ldots,\left(\mathbf{H}_{28}\right)_{k b a}$ are listed in the Appendix.

According to Eqs. (17) and (18), the entries of the Jacobian formulation of the additional inertial force are derived as follows:

when $k=1,2, \ldots, 36$ and $l=1,2, \ldots, 36$,

$$
\begin{aligned}
\frac{\partial F_{k}^{a}}{\partial q_{l}}= & \dot{m}_{1}^{2}\left(\mathbf{H}_{5}\right)_{k l}+\dot{n}_{1}^{2}\left(\mathbf{H}_{6}\right)_{k l}+\dot{m}_{2}^{2}\left(\mathbf{H}_{7}\right)_{k l}+\dot{n}_{2}^{2}\left(\mathbf{H}_{8}\right)_{k l} \\
& +\dot{m}_{1} \dot{n}_{1}\left(\mathbf{H}_{9}\right)_{k l}+\dot{m}_{1} \dot{m}_{2}\left(\mathbf{H}_{10}\right)_{k l}+\dot{m}_{1} \dot{n}_{2}\left(\mathbf{H}_{11}\right)_{k l} \\
& +\dot{n}_{1} \dot{m}_{2}\left(\mathbf{H}_{12}\right)_{k l}+\dot{n}_{1} \dot{n}_{2}\left(\mathbf{H}_{13}\right)_{k l}+\dot{m}_{2} \dot{n}_{2}\left(\mathbf{H}_{14}\right)_{k l},
\end{aligned}
$$

when $k=1,2, \ldots, 36$ and $l=37,38, \ldots, 40$,

$$
\begin{aligned}
\frac{\partial F_{k}^{a}}{\partial q_{l}} & =\left[\dot{m}_{1}\left(\mathbf{H}_{1}\right)_{k a, q_{l}}+\dot{n}_{1}\left(\mathbf{H}_{2}\right)_{k a, q_{l}}+\dot{m}_{2}\left(\mathbf{H}_{3}\right)_{k a, q_{l}}+\dot{n}_{2}\left(\mathbf{H}_{4}\right)_{k a, q_{l}}\right] \dot{q}_{a} \\
& +\left[\dot{m}_{1}^{2}\left(\mathbf{H}_{5}\right)_{k a, q_{l}}+\dot{n}_{1}^{2}\left(\mathbf{H}_{6}\right)_{k a, q_{l}}+\dot{m}_{2}^{2}\left(\mathbf{H}_{7}\right)_{k a, q_{l}}+\dot{n}_{2}^{2}\left(\mathbf{H}_{8}\right)_{k a, q_{l}}\right] q_{a} \\
& +\left[\dot{m}_{1} \dot{n}_{1}\left(\mathbf{H}_{9}\right)_{k a, q_{l}}+\dot{m}_{1} \dot{m}_{2}\left(\mathbf{H}_{10}\right)_{k a, q_{l}}+\dot{m}_{1} \dot{n}_{2}\left(\mathbf{H}_{11}\right)_{k a, q_{l}}\right] q_{a} \\
& +\left[\dot{n}_{1} \dot{m}_{2}\left(\mathbf{H}_{12}\right)_{k a, q_{l}}+\dot{n}_{1} \dot{n}_{2}\left(\mathbf{H}_{13}\right)_{k a, q_{l}}+\dot{m}_{2} \dot{n}_{2}\left(\mathbf{H}_{14}\right)_{k a, q_{l}}\right] q_{a},
\end{aligned}
$$

when $k=37,38, \ldots, 40$ and $l=1,2, \ldots, 36$,

$$
\begin{aligned}
\frac{\partial F_{k}^{a}}{\partial q_{l}}= & {\left[\dot{m}_{1}\left(\mathbf{H}_{15}\right)_{k l a}+\dot{n}_{1}\left(\mathbf{H}_{16}\right)_{k l a}+\dot{m}_{2}\left(\mathbf{H}_{17}\right)_{k l a}+\dot{n}_{2}\left(\mathbf{H}_{18}\right)_{k l a}\right] \dot{q}_{a} } \\
& +\left[\dot{m}_{1}^{2}\left(\mathbf{H}_{19}\right)_{k l a}+\dot{n}_{1}^{2}\left(\mathbf{H}_{20}\right)_{k l a}+\dot{m}_{2}^{2}\left(\mathbf{H}_{21}\right)_{k l a}+\dot{n}_{2}^{2}\left(\mathbf{H}_{22}\right)_{k l a}\right] q_{a} \\
& +\left[\dot{m}_{1}^{2}\left(\mathbf{H}_{19}\right)_{k a l}+\dot{n}_{1}^{2}\left(\mathbf{H}_{20}\right)_{k a l}+\dot{m}_{2}^{2}\left(\mathbf{H}_{21}\right)_{k a l}+\dot{n}_{2}^{2}\left(\mathbf{H}_{22}\right)_{k a l}\right] q_{a} \\
& +\left[\dot{m}_{1} \dot{n}_{1}\left(\mathbf{H}_{23}\right)_{k l a}+\dot{m}_{1} \dot{m}_{2}\left(\mathbf{H}_{24}\right)_{k l a}+\dot{m}_{1} \dot{n}_{2}\left(\mathbf{H}_{25}\right)_{k l a}\right] q_{a} \\
& +\left[\dot{m}_{1} \dot{n}_{1}\left(\mathbf{H}_{23}\right)_{k a l}+\dot{m}_{1} \dot{m}_{2}\left(\mathbf{H}_{24}\right)_{k a l}+\dot{m}_{1} \dot{n}_{2}\left(\mathbf{H}_{25}\right)_{k a l}\right] q_{a} \\
& +\left[\dot{n}_{1} \dot{m}_{2}\left(\mathbf{H}_{26}\right)_{k l a}+\dot{n}_{1} \dot{n}_{2}\left(\mathbf{H}_{27}\right)_{k l a}+\dot{m}_{2} \dot{n}_{2}\left(\mathbf{H}_{28}\right)_{k l a}\right] q_{a} \\
& +\left[\dot{n}_{1} \dot{m}_{2}\left(\mathbf{H}_{26}\right)_{k a l}+\dot{n}_{1} \dot{n}_{2}\left(\mathbf{H}_{27}\right)_{k a l}+\dot{m}_{2} \dot{n}_{2}\left(\mathbf{H}_{28}\right)_{k a l}\right] q_{a},
\end{aligned}
$$

when $k=37,38, \ldots, 40$ and $l=37,38, \ldots, 40$, 


$$
\begin{aligned}
\frac{\partial F_{k}^{a}}{\partial q_{l}} & =\left[\dot{m}_{1}\left(\mathbf{H}_{15}\right)_{k b a, q_{l}}+\dot{n}_{1}\left(\mathbf{H}_{16}\right)_{k b a, q_{l}}+\dot{m}_{2}\left(\mathbf{H}_{17}\right)_{k b a, q_{l}}+\dot{n}_{2}\left(\mathbf{H}_{18}\right)_{k b a, q_{l}}\right] q_{b} \dot{q}_{a} \\
& +\left[\dot{m}_{1}^{2}\left(\mathbf{H}_{19}\right)_{k b a, q_{l}}+\dot{n}_{1}^{2}\left(\mathbf{H}_{20}\right)_{k b a, q_{l}}+\dot{m}_{2}^{2}\left(\mathbf{H}_{21}\right)_{k b a, q_{l}}+\dot{n}_{2}^{2}\left(\mathbf{H}_{22}\right)_{k b a, q_{l}}\right] q_{b} q_{a} \\
& +\left[\dot{m}_{1} \dot{n}_{1}\left(\mathbf{H}_{23}\right)_{k b a, q_{l}}+\dot{m}_{1} \dot{m}_{2}\left(\mathbf{H}_{24}\right)_{k b a, q_{l}}+\dot{m}_{1} \dot{n}_{2}\left(\mathbf{H}_{25}\right)_{k b a, q_{l}}\right] q_{b} q_{a} \\
& +\left[\dot{n}_{1} \dot{m}_{2}\left(\mathbf{H}_{26}\right)_{k b a, q_{l}}+\dot{n}_{1} \dot{n}_{2}\left(\mathbf{H}_{27}\right)_{k b a, q_{l}}+\dot{m}_{2} \dot{n}_{2}\left(\mathbf{H}_{28}\right)_{k b a, q_{l}}\right] q_{b} q_{a} .
\end{aligned}
$$

In Eqs. (19)-(22), the subscripts $a, b=1,2, \ldots, 36$ and $\left(\mathbf{H}_{1}\right)_{k a, q_{l}}$ represents the partial derivatives of $\left(\mathbf{H}_{1}\right)_{k a}$ with respect to the local material coordinates $q_{l}(l=37,38, \ldots, 40)$. The detailed expressions of $\left(\mathbf{H}_{1}\right)_{k a, q_{l}},\left(\mathbf{H}_{2}\right)_{k a, q_{l}}, \ldots,\left(\mathbf{H}_{14}\right)_{k a, q_{l}}$ and $\left(\mathbf{H}_{15}\right)_{k b a, q_{l}},\left(\mathbf{H}_{16}\right)_{k b a, q_{l}}, \ldots,\left(\mathbf{H}_{28}\right)_{k b a, q_{l}}$ are listed in the Appendix.

2.4 Time integration scheme considering variable-length bodies

The dynamic equation of an FMBS modeled via ALE-ANCF can be expressed as [26]

$$
\left\{\begin{array}{l}
\mathbf{M}(t) \ddot{\mathbf{q}}_{D}+\mathbf{\Phi}_{, \mathbf{q}_{D}}^{\mathrm{T}} \lambda+\mathbf{F}^{e}+\mathbf{F}^{a}=\mathbf{Q} \\
\mathbf{\Phi}\left(\mathbf{q}_{D}, t\right)=\mathbf{0}
\end{array}\right.
$$

where $\mathbf{M}(t)=\int_{V_{0}} \rho \mathbf{N}^{\mathrm{T}} \mathbf{N} d V$ is the time-dependent mass matrix of the system, $\mathbf{q}_{D}$ is the generalized coordinate vector of the system, $\boldsymbol{\Phi}_{\mathbf{q}_{D}}$ is the Jacobian of the constraint $\Phi$ and $\lambda$ is the Lagrange multiplier vector. $\mathbf{F}^{e}$ and $\mathbf{F}^{a}$ are the elastic force vector assembled by Eq. (9) and the additional inertial force vector assembled by Eqs. (17) and (18), respectively. $\mathbf{Q}$ denotes the generalized external force vector. The second equation in Eq. (23) represents the kinematic constraints including driving constraints.

To solve the dynamic equation in Eq. (23), the generalized- $\alpha$ method [42-45] is utilized and reevaluated to deal with the variation of the degrees of freedom due to the variable-length. Fig. 3 gives the generalized- $\alpha$ time integration scheme considering variable-length bodies. The readers are referred to Refs. [42-45] for detailed descriptions of the generalized- $\alpha$ method. The key point of the time integration scheme in Fig. 3 is that when the length of a flexible body is varying, ALE-ANCF nodes of boundary elements have to be inserted or removed to avoid excessively long or short lengths of boundary elements. Excessively long lengths of boundary elements can reduce the accuracy of the computational result, while excessively short case can result 
in the singularity of the stiffness matrix. Therefore, in Fig. 3, when the length of the boundary element $L_{b}$ is longer than the given maximum length $L_{\max }$, new nodes are inserted into the boundary element, as illustrated in Fig. 4(a). Likewise, when the length of the boundary element $L_{b}$ is shorter than the given minimum length $L_{\mathrm{min}}$, the nodes inside the boundary element are removed, as illustrated in Fig. 4(b).

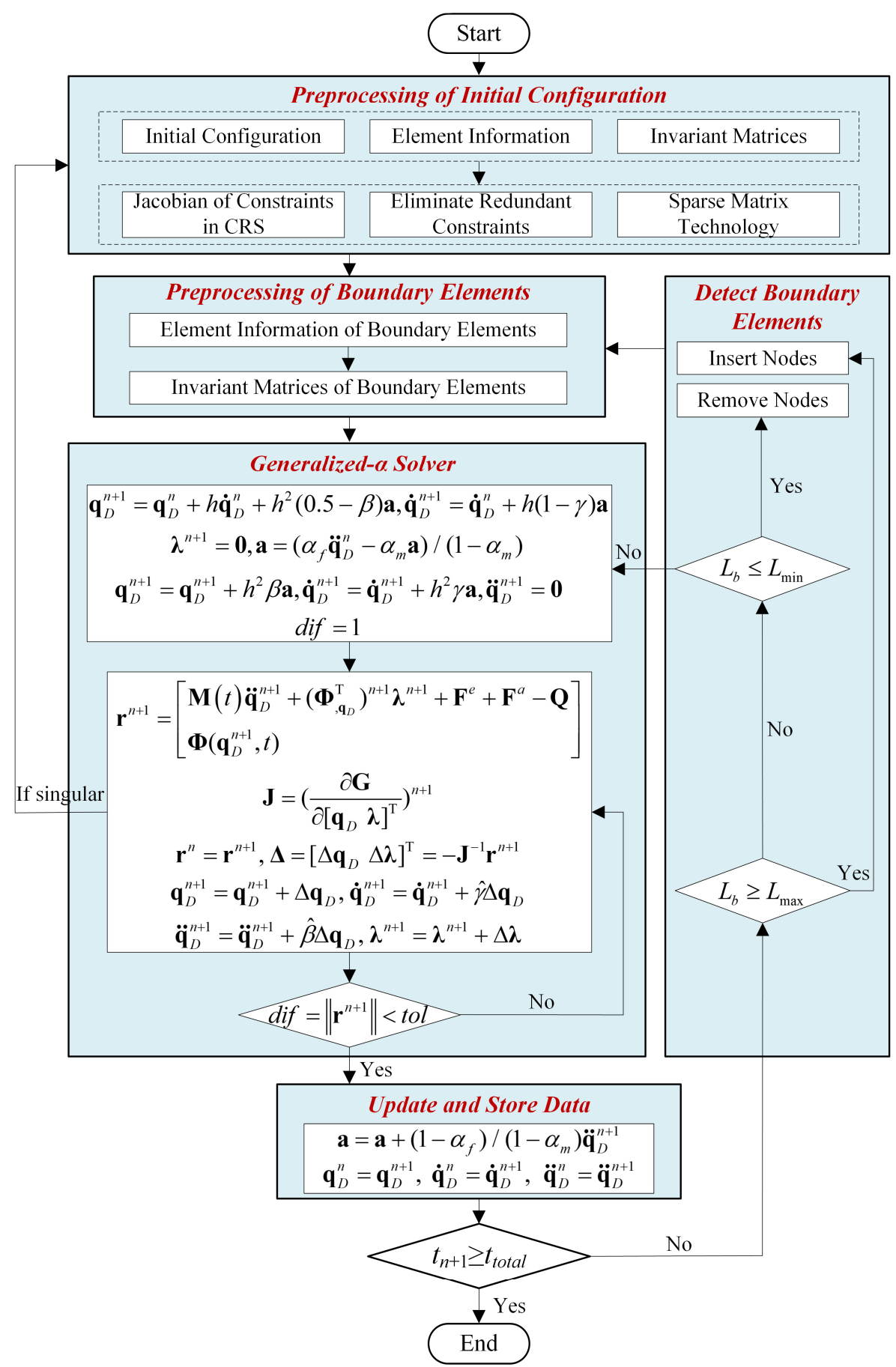

Fig. 3 Time integration scheme considering variable-length bodies 


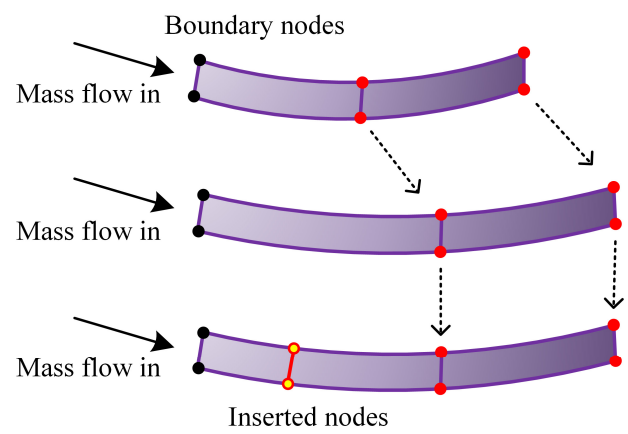

(a) Insert nodes

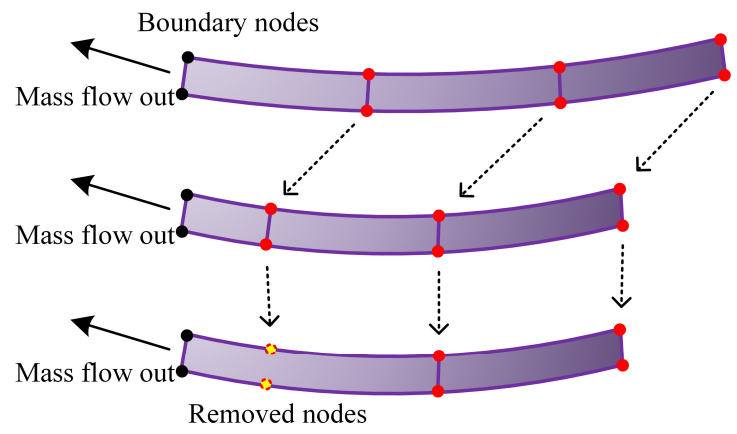

(b) Remove nodes

Fig. 4 Illustration of boundary element treatment

\section{Topology optimization of an FMBS}

\subsection{Moving morphable components based topology optimization}

In the MMC-based topology optimization method, the structural topology is first represented by a set of morphable components. The optimal topology, then, can be obtained by optimizing the layout including positions and shapes of those components [34-37]. To achieve this goal, the structural components have to be explicitly and mathematically described by the topology description function (TDF). Fig. 5 depicts the iteration history of the MMC-based topology optimization process, where the components in different colors are of the same kind for illustration purpose.
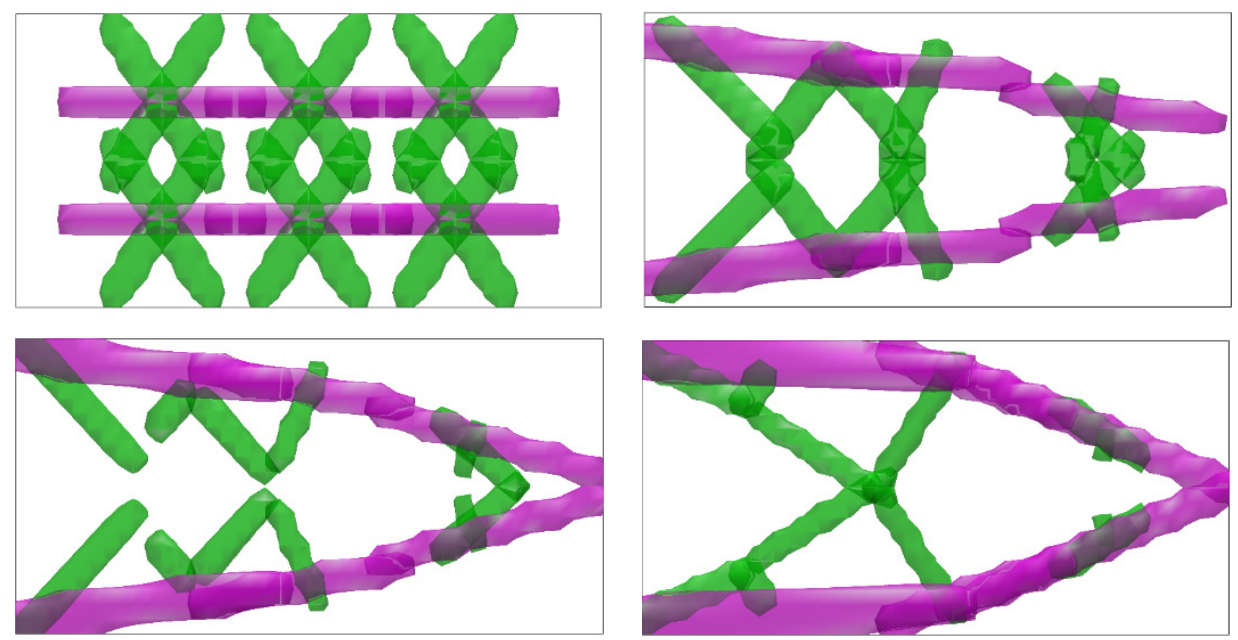

Fig. 5 Iteration history of the MMC-based topology optimization process 


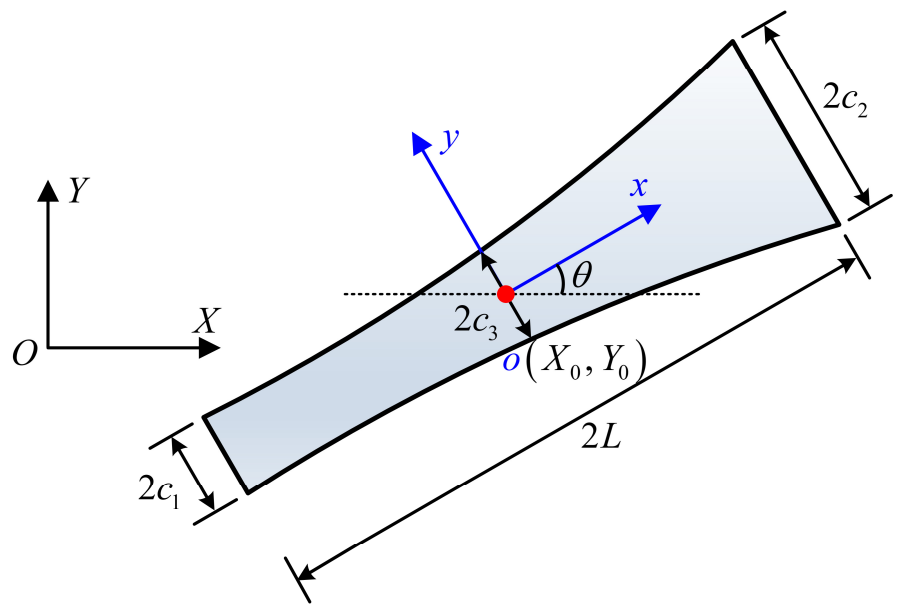

(a) Schematic diagram

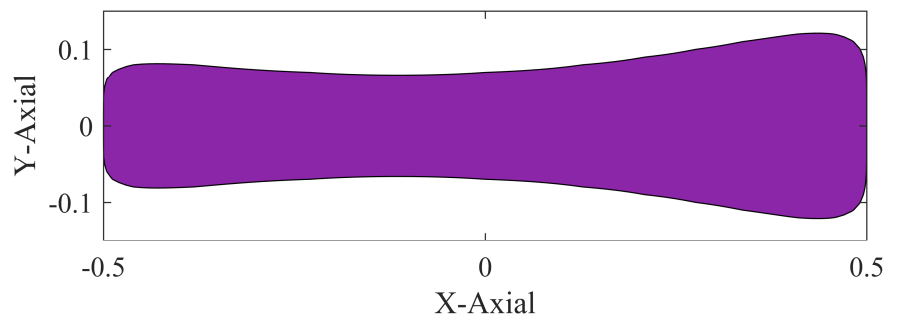

(b) TDF with $L=0.5, c_{1}=0.1, c_{2}=0.15$ and $c_{3}=0.07$

Fig. 6 A thickness-varying structural component

In this study, the following TDF is employed to describe the geometry of a planar thickness-varying structural component, as shown in Fig. 6(a) [34]

$$
\chi(X, Y)=1-\left(\frac{x}{L}\right)^{p}-\left(\frac{y}{g(x)}\right)^{p},
$$

where

$$
\left[\begin{array}{l}
x \\
y
\end{array}\right]=\left[\begin{array}{cc}
\cos \theta & \sin \theta \\
-\sin \theta & \cos \theta
\end{array}\right]\left[\begin{array}{c}
X-X_{0} \\
Y-Y_{0}
\end{array}\right] .
$$

In Eq. (24) and (25), $p$ is a large even integer number (usually $p=6$ ), $O-X Y$ and $o-x y$ denote the global frame of coordinates and the local frame of coordinates, respectively. $\left(X_{0}, Y_{0}\right)$ represents the global coordinates of the component center. $L, g(x)$ and $\theta$ are the semi-length, the thickness profile and the inclined angle with respect to $O X$ axis of the component, respectively. Here, the thickness profile yields the quadratic relation as follows

$$
g(x)=\frac{c_{1}+c_{2}-2 c_{3}}{2 L^{2}} x^{2}+\frac{c_{2}-c_{1}}{2 L} x+c_{3},
$$

where the parameters $c_{1}, c_{2}$ and $c_{3}$ are the semi-thickness parameters at both ends 
and in the middle of the structural component, as illustrated in Fig. 6(a). Fig. 6(b) gives the TDF of a thickness-varying structural component with $L=0.5, c_{1}=0.1, c_{2}=$ 0.15 and $c_{3}=0.07$.

According to Eqs. (24)-(26), the design variable vector of the component is defined as follows

$$
\mathbf{b}=\left[\begin{array}{lllllll}
X_{0} & Y_{0} & L & \theta & c_{1} & c_{2} & c_{3}
\end{array}\right]^{\mathrm{T}} .
$$

For a single component, the material domain $\Omega_{i}$, the material interface $\partial \Omega_{i}$ of the $i$ th component and the void domain $D \backslash \Omega_{i}$ can be represented by the TDF as

$$
\left\{\begin{array}{l}
\chi_{i}(\mathbf{X})>0 \Leftrightarrow \mathbf{X} \in \Omega_{i} \\
\chi_{i}(\mathbf{X})=0 \Leftrightarrow \mathbf{X} \in \partial \Omega_{i}, \\
\chi_{i}(\mathbf{X})<0 \Leftrightarrow \mathbf{X} \in D \backslash \Omega_{i}
\end{array}\right.
$$

where $\mathbf{X}$ is an arbitrary point in the design domain $D$ of $\mathbb{R}^{2}, \chi_{i}$ is the TDF defined in Eq. (24) for the $i$-th component and $N$ is the total number of structural components. Then, the topology description of the entire design domain can be achieved by defining the TDF for all the structural components as follows

$$
\left\{\begin{array}{l}
\chi(\mathbf{X})>0 \Leftrightarrow \mathbf{X} \in \Omega \\
\chi(\mathbf{X})=0 \Leftrightarrow \mathbf{X} \in \partial \Omega \\
\chi(\mathbf{X})<0 \Leftrightarrow \mathbf{X} \in D \backslash \Omega
\end{array}\right.
$$

where $\chi=\max \left(\chi_{1}, \cdots, \chi_{N}\right)$ and $\Omega=\bigcup_{i=1}^{N} \Omega_{i}$ represents the material domain occupied by at least one structural component.

3.2 Topology optimization formulation

The formulation of the MMC-based topology optimization for an FMBS with variable-length bodies can be mathematically expressed as

$$
\begin{array}{ll}
\text { find } & \mathbf{b} \\
\text { to minimize } & f\left(\mathbf{b}, \mathbf{q}_{D}(t)\right) \\
\text { subject to } & \mathbf{M}(\mathbf{b}) \ddot{\mathbf{q}}_{D}+\boldsymbol{\Phi}_{, \mathbf{q}_{D}}^{\mathrm{T}} \lambda+\mathbf{F}^{e}(\mathbf{b})+\mathbf{F}^{a}(\mathbf{b})=\mathbf{Q} \\
& \boldsymbol{\Phi}\left(\mathbf{q}_{D}, t\right)=0 \\
& V(\mathbf{b})-V_{\max } \leq 0 \\
& \mathbf{b} \subset u_{\mathbf{b}} .
\end{array}
$$

In Eq. (30), $\mathbf{b}=\left[\begin{array}{lllll}\mathbf{b}_{1}^{\mathrm{T}} & \cdots & \mathbf{b}_{i}^{\mathrm{T}} & \cdots & \mathbf{b}_{N}^{\mathrm{T}}\end{array}\right]^{\mathrm{T}}$ is the vector of design variables with 
$\mathbf{b}_{i}$ denoting the vector of design variables of the $i$-th structural component in Eq. (27). From Eq. (30), it can be easily found that $\mathbf{b}$ belongs to an admissible set $u_{\mathbf{b}}$. As a result, the minimum thickness of the components can be controlled by setting the lower bounds of the geometric design variables in $u_{\mathbf{b}}$. Other symbols in Eq. (30) are explained as follows. $f\left(\mathbf{b}, \mathbf{q}_{D}(t)\right)$ is the objective function associated with the design variables and time $t$ for the dynamic optimization problem. Taking the stiffness design into consideration, the objective function is defined as the mean compliance of the flexible bodies. The first two equations in the constraints represent the dynamic equation of an FMBS, which have been explained in detail in section 2.3. $V-V_{\max } \leq 0$ is called the inequality volume constraint, where $V$ is the volume ratio of the flexible bodies to be optimized and $V_{\max }$ is the volume fraction determined by the designers.

\subsection{ESL-based computation flow of optimization}

In solving the topology optimization for the dynamic response of an FMBS as shown in Eq. (30), the ESL method has seen successful applications [15-18, 46]. Compared with the integrated optimization method [32], the ESL method has many merits, such as high efficiency in computation and convenience in implementation [15, 32]. In this study, the ESL method is modified to deal with the topology optimization problem for the dynamic response of an FMBS with variable-length bodies.

Based on the ANCF, the ESL sets are defined as the static loads that generate the same deformations of the flexible bodies in the static analysis as those from the nonlinear dynamic analysis [15], that is,

$$
\mathbf{F}_{e q}\left(t_{i}\right)=\mathbf{K}\left(\mathbf{b}, \mathbf{q}_{D}\left(t_{i}\right)\right)\left(\mathbf{q}_{D}\left(t_{i}\right)-\mathbf{q}_{R}\left(t_{i}\right)\right) \quad(i=1,2, \ldots, n),
$$

where $\mathbf{K}$ is the nonlinear stiffness matrix of ANCF associated with the design variable vector $\mathbf{b}$ and the generalized nodal coordinate vector $\mathbf{q}_{D}, n$ is the total number of time steps concerned and $t_{i}$ represents the simulation time at $i$-th time step. The generalized coordinate vectors $\mathbf{q}_{D}$ and $\mathbf{q}_{R}$ are obtained from the dynamic analysis and the simulation of rigid multibody system, respectively.

However, some challenges arise when computing the ESL sets for an FMBS modeled via ALE-ANCF as the lengths of the flexible bodies to be optimized are varying over time. As a consequence, the design domains are different at different time steps. To overcome the difficulty in computing ESL sets and to obtain a comprehensive 
optimization result, the concept of virtual design domain is proposed in this study. As illustrated in Fig. 7, for the extension case of a flexible body, the stretching domain is called the actual design domain $D^{a}$, and the unstretched domain is the so-called virtual design domain $D^{v}$. Likewise, for the contraction case, the contracted domain is named the virtual design domain $D^{v}$. The whole design domain of the flexible body, hence, can be defined as $D=D^{a} \cup D^{v}$. The difference between $D^{a}$ and $D^{v}$ is that the ESL sets in the two domains are separately defined as follows

$$
\mathbf{F}_{e q}\left(\mathbf{X}, t_{i}\right)=\left\{\begin{array}{cc}
\mathbf{K}^{a}\left(\mathbf{b}, \mathbf{q}_{D}^{a}\left(t_{i}\right)\right)\left(\mathbf{q}_{D}^{a}\left(t_{i}\right)-\mathbf{q}_{R}^{a}\left(t_{i}\right)\right) & \left(\mathbf{X} \in D^{a}\right) \\
\mathbf{0} & \left(\mathbf{X} \in D^{v}\right)
\end{array} \quad(i=1,2, \ldots, n) .\right.
$$

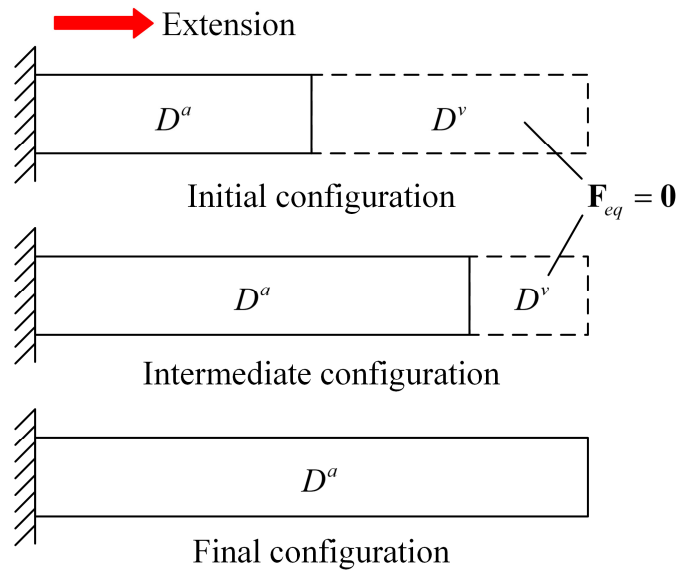

(a) Extension in length

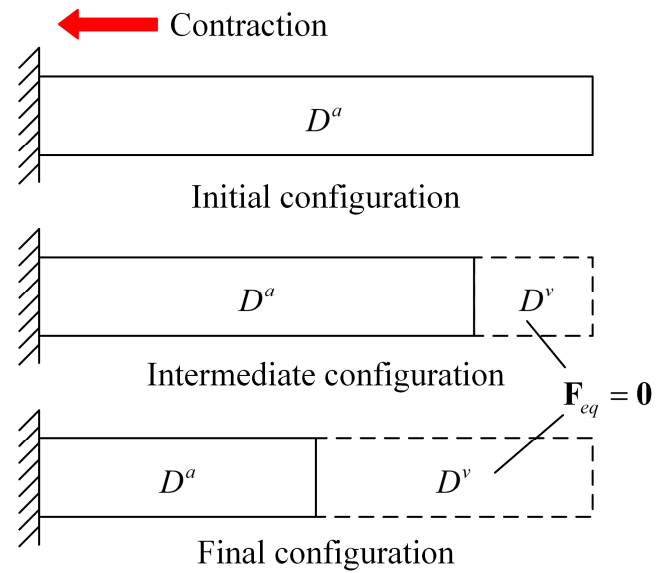

(b) Contraction in length

Fig. 7 The concept of virtual design domain

In Eq. (32), the ESL sets are computed in the same way as that in Eq. (31) in the actual design domain $D^{a}$, and are identically equal to zeros in the virtual design domain $D^{v}$. However, $\mathbf{K}^{a}, \mathbf{q}_{D}^{a}$ and $\mathbf{q}_{R}^{a}$ denoting the nonlinear stiffness matrix and generalized coordinates in the actual design domain $D^{a}$, are different from $\mathbf{K}, \mathbf{q}_{D}$ and $\mathbf{q}_{R}$ in Eq. (31).

According to Eq. (32), when $\mathbf{F}_{e q}$ is used as the external force vector, the generalized coordinate vector $\mathbf{q}_{s}$ can be obtained from the static analysis as follows

$$
\mathbf{K}\left(\mathbf{b}, \mathbf{q}_{D}\left(t_{i}\right)\right)\left(\mathbf{q}_{s}\left(t_{i}\right)-\mathbf{q}_{R}\left(t_{i}\right)\right)=\mathbf{F}_{e q}\left(\mathbf{X}, t_{i}\right) \quad(i=1,2, \ldots, n),
$$

where $\mathbf{K}$ is the stiffness matrix defined in the whole design domain $D$. If only single flexible body is considered, the equilibrium of Eq. (33) can be directly solved by taking 
the displacement boundary conditions into account. Otherwise, if more than one flexible body is considered, the deformation of each body has to be computed independently to get rid of the impact of the kinematic joints [27].

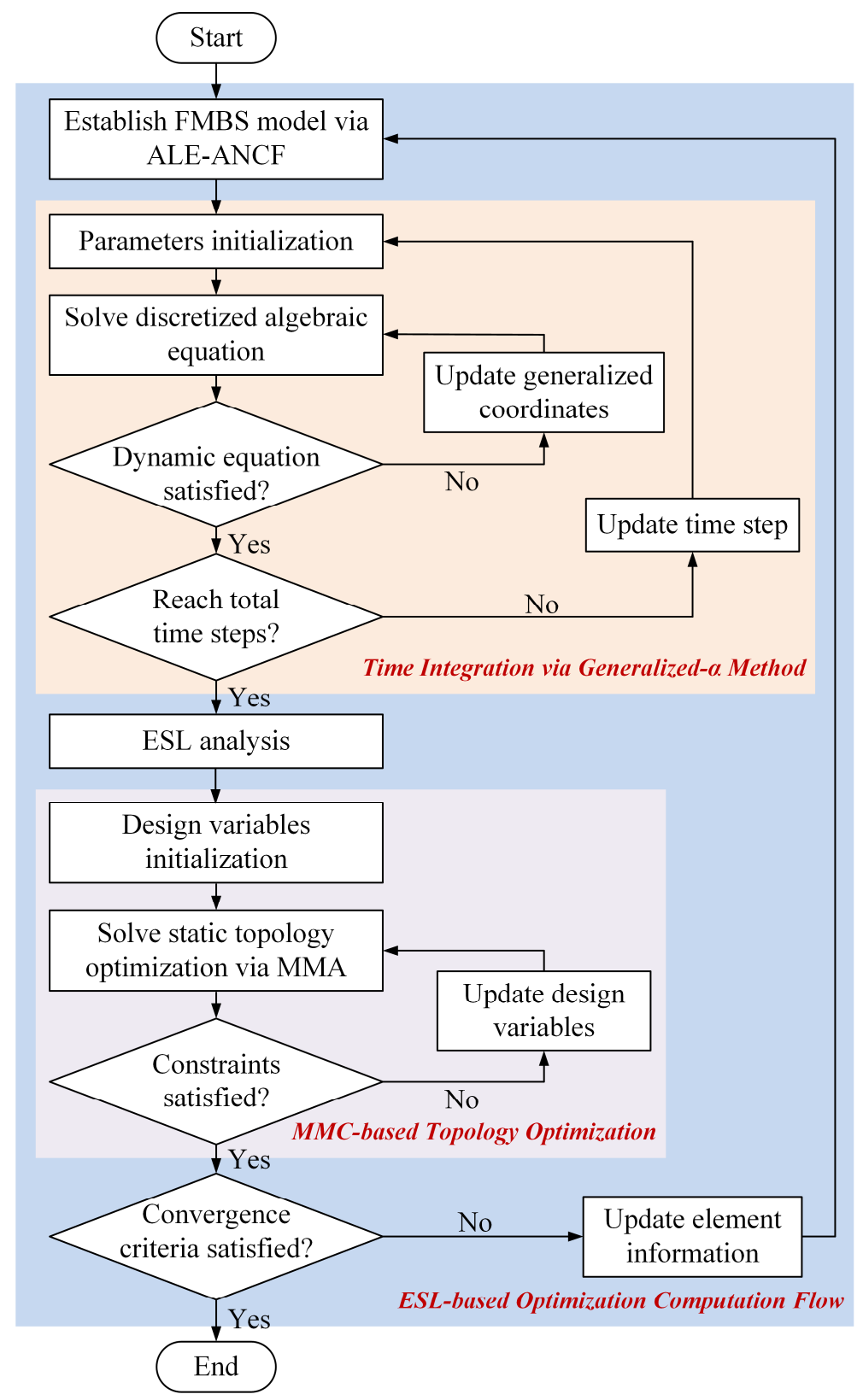

Fig. 8 The flowchart of ESL-based computation for the topology optimization of an FMBS with variable-length bodies

To this end, the topology optimization for the dynamic response of an FMBS with variable-length bodies as shown in Eq. (30) can be turned into a static one under multiple loading conditions in the following way 


$$
\begin{array}{ll}
\text { find } & \mathbf{b} \\
\text { to minimize } & f\left(\mathbf{b}, \mathbf{q}_{S}(t)\right) \\
\text { subject to } & \mathbf{K}\left(\mathbf{b}, \mathbf{q}_{D}\left(t_{i}\right)\right)\left(\mathbf{q}_{S}\left(t_{i}\right)-\mathbf{q}_{R}\left(t_{i}\right)\right)=\mathbf{F}_{e q}\left(\mathbf{X}, t_{i}\right) \\
& (i=1,2, \ldots, n) \\
& V(\mathbf{b})-V_{\max } \leq 0 \\
& \mathbf{b} \subset u_{\mathbf{b}}
\end{array}
$$

In Eq. (34), $f$ is the objection function, defined as the weighted summation of the compliances of the flexible bodies at all time steps, namely,

$$
f=\sum_{i=1}^{n} w_{i}\left(\boldsymbol{\sigma}_{i}\left(\mathbf{q}_{s}\right)^{\mathrm{T}} \boldsymbol{\varepsilon}_{i}\left(\mathbf{q}_{S}\right)\right)
$$

where $w_{i}$, determined by the expert evaluation method of weights $[15,47]$, is the weight for the $i$-th time step, $\boldsymbol{\sigma}_{i}$ and $\boldsymbol{\varepsilon}_{i}$ are the Piola-Kirchhoff stress tensor of the second kind and the Green-Lagrange strain tensor at the $i$-th time step, respectively. To solve the topology optimization problem of static response in Eq. (34), the method of moving asymptotes (MMA) [34, 48] is employed. Then, the whole ESL-based computation flow is briefly illustrated in Fig. 8 for the topology optimization of an FMBS with variable-length bodies.

\section{Numerical examples}

In this section, three numerical examples are presented to validate the accuracy of the thin plate element of ALE-ANCF and the topology optimization approach proposed for an FMBS with variable-length bodies. The first example is a thin plate pendulum falling down under gravity [26]. The second one is the topology optimization for a rotating variable-length plate with different extension velocities taken into account and the third one is the topology optimization of a 3 degrees-of-freedom (DOF) motion platform. In the first example, the elastic force vector of the thin plate element of ALEANCF is computed according to Eq. (9) with the out-of-plane strain energy taken into account. For efficient computation, in the second and third ones, the elastic force vector is only computed by Eq. (10).

\subsection{Dynamics of a thin plate pendulum}

This classic example has been widely studied [26, 39, 49-53] to validate different 
kinds of plate and shell elements of ANCF. Compared with the fully parameterized elements of ANCF, the gradient deficient thin plate element of ANCF exhibits better computational efficiency and convergence rate [39]. Besides, many methods have been proposed to solve the locking problems of ANCF $[40,54,55]$. As a thin plate element of ALE-ANCF can be degenerated into a thin plate element of ANCF when the four material coordinates of the element are fixed, the results obtained from the two kinds of finite element of ANCF are comparatively studied. As shown in Fig. 9, the pendulum is a thin square plate [26] with an edge length of $0.3 \mathrm{~m}$ and a thickness of $0.01 \mathrm{~m}$. The mass density of the plate is $7810 \mathrm{~kg} / \mathrm{m}^{3}$, and Young's modulus and Poison's ratio are $1 \times 10^{5} \mathrm{~Pa}$ and 0.3 , respectively. The pendulum is initially at rest as shown in Fig. 9, where a corner point of the plate is fixed via a spherical joint at the origin $O$ and starts falling down due to gravity when $t>0$. To validate the thin plate element of ALEANCF derived in Section 2, the pendulum is modeled via $8 \times 8$ thin plate elements of ALE-ANCF in this study and $8 \times 8$ traditional thin plate elements of ANCF [38], respectively.

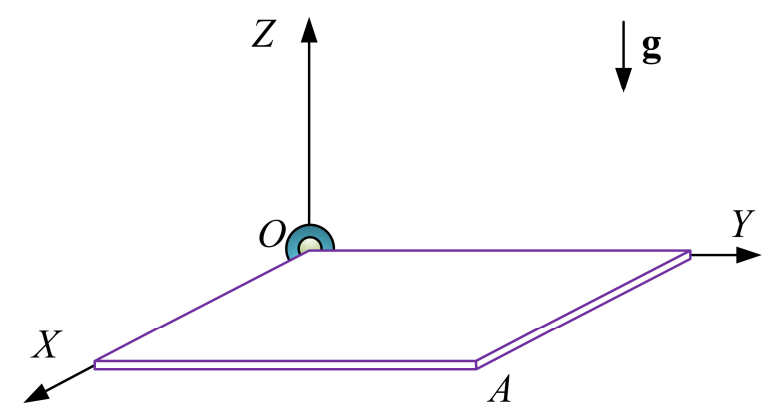

Fig. 9 A thin plate pendulum under gravity

Fig. 10 illustrates the deformed shapes of the pendulum at four moments, and shows good agreement between the ALE-ANCF model and ANCF model. Fig. 11 gives the comparison results of the $x$-, $y$ - and $z$-displacements of corner point $A$, and shows that the results from ALE-ANCF model and ANCF model match with each other very well. Fig. 12 presents the energy of the pendulum with an increase of time. Fig. 12(a) indicates that the total energy of the pendulum keeps constant since the only external force comes from gravity. Fig. 12(b) depicts the perfect agreement of the strain energy between the ALE-ANCF and ANCF models, as well as between the results in this study and those in Ref. [26]. Hence, it is possible to reach the assertion that the thin plate element of ALE-ANCF proposed here can accurately describe an FMBS subject to both large overall motion and large deformation. 


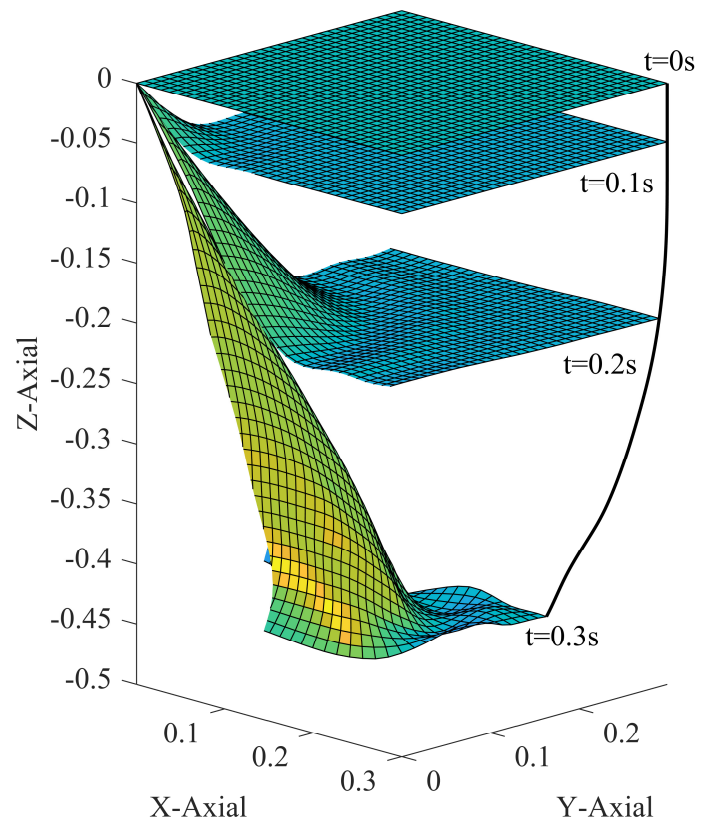

(a) $8 \times 8$ thin plate elements of ALE-ANCF

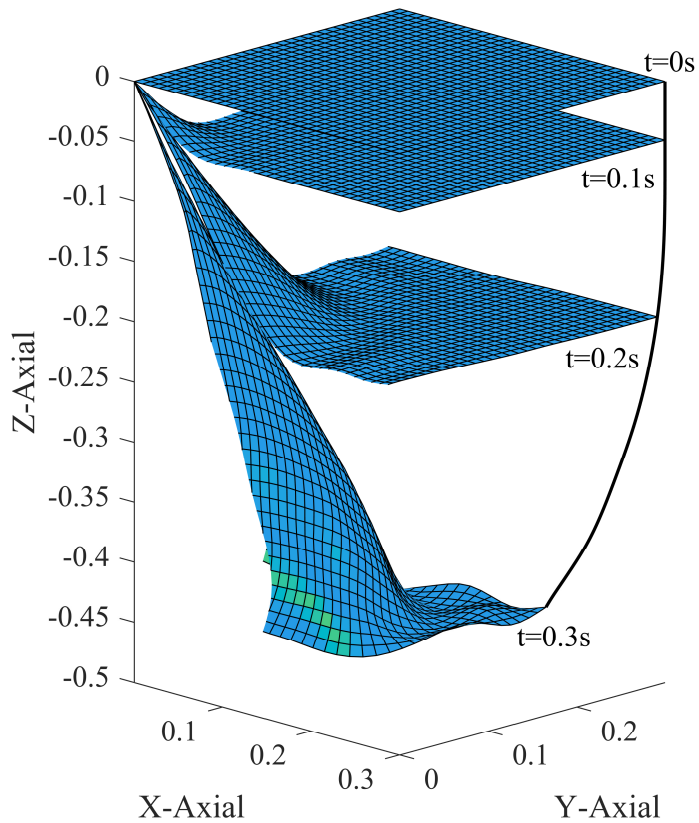

(b) $8 \times 8$ thin plate elements of $\mathrm{ANCF}$

Fig. 10 Comparison of the deformed shapes of the thin plate pendulum

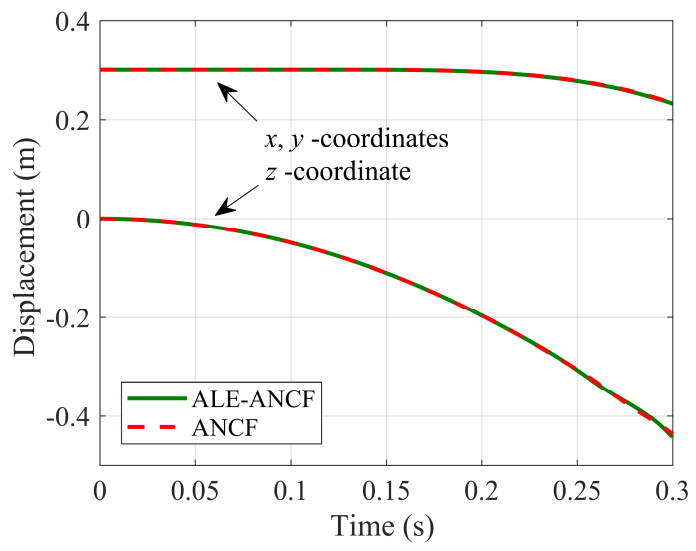

Fig. 11 Comparison of two models for predicting the displacement of corner point $A$

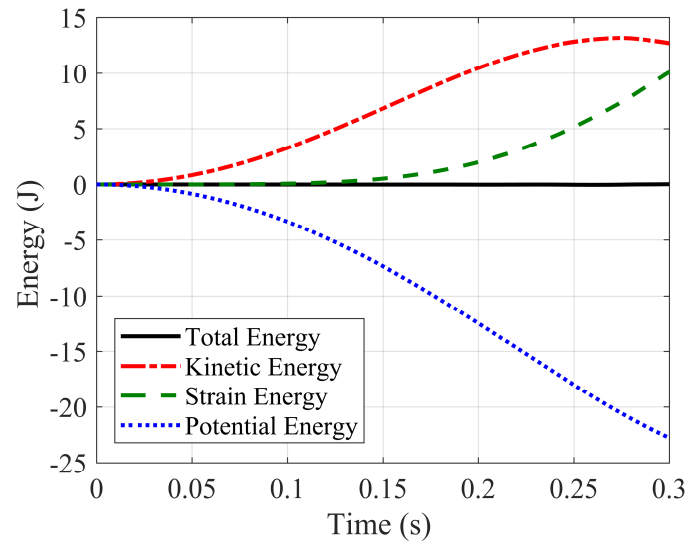

(a) Energy balance of ALE-ANCF model

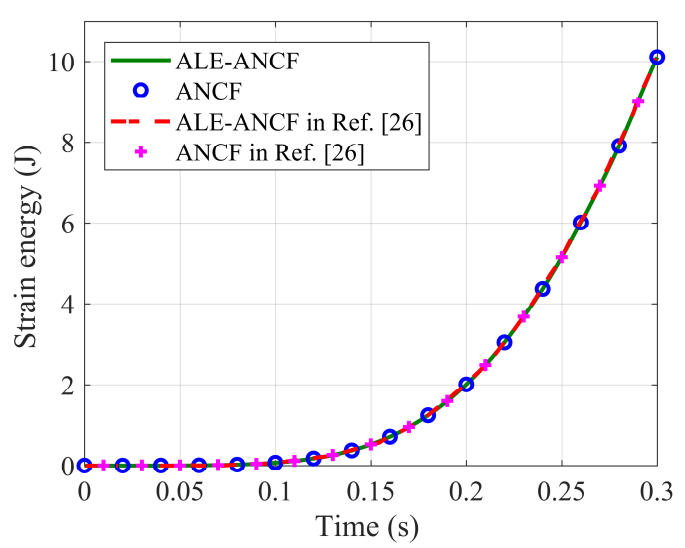

(b) Comparison of strain energy

Fig. 12 Energy of the thin plate pendulum with respect to time 


\subsection{Topology optimization of a rotating variable-length plate}

This example simplifies the four deployable beams of a spinning solar sail [6], as shown in Fig. 1, to a rotating variable-length plate carrying a tip mass. Due to the high efficiency of the adopted MMC methods, the proposed optimization method can be further used to optimize the deployable beams and membranes in a large-scale space structure. As shown in Fig. 13, the rotating thin plate is connected to the ground via a revolute joint at origin $O$, and a tip mass of $0.5 \mathrm{~kg}$ is mounted at the free end of the plate. The rotating plate has an initial length of $0.5 \mathrm{~m}$, a width of $0.1 \mathrm{~m}$ and a thickness of $0.01 \mathrm{~m}$. The mass density of the plate is $2700 \mathrm{~kg} / \mathrm{m}^{3}$, and Young's modulus and Poison's ratio are $1 \times 10^{8} \mathrm{~Pa}$ and 0.3 , respectively. The plate moves in the $X-Y$ plane at a constant angular velocity $\omega=2 \pi \mathrm{rad} / \mathrm{s}$ while its length extends at the same time. The initial configuration of the rotating plate is modeled via $10 \times 2$ thin plate elements of ALE-ACNF. The maximum length of the boundary element is $0.1 \mathrm{~m}$. That is, when the length of the boundary element reaches $0.1 \mathrm{~m}$, two nodes of ALE-ANCF are inserted to divide the boundary element from the middle into two elements, as illustrated in Fig. $4(a)$.

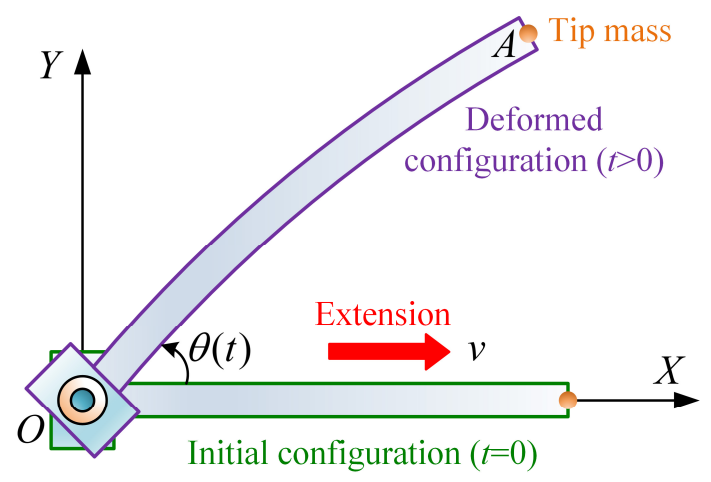

Fig. 13 A rotating variable-length plate

Two kinds of extension velocities are investigated to topologically optimize the rotating plate, that is, $v=0.1 \mathrm{~m} / \mathrm{s}$ and $v=0.5 \mathrm{~m} / \mathrm{s}$. The analysis time is set to be $1 \mathrm{~s}$ in a period of motion. Therefore, the final lengths for the two extension velocity cases are $0.6 \mathrm{~m}$ and $1.0 \mathrm{~m}$. To compute the ESL sets, the time step size is set as $0.05 \mathrm{~s}$ for the extension velocity $0.1 \mathrm{~m} / \mathrm{s}$ and $0.01 \mathrm{~s}$ for the extension velocity $0.5 \mathrm{~m} / \mathrm{s}$. Hence, there are 20 and 100 time steps all together correspondingly. During the static topology optimization, the weights for the 20 and 100 time steps can be determined via an expert evaluation method of weights to avoid the subjective influence of the designers. 
However, the study does not focus on the influence of the weights. To take the practical situation into consideration, all the weights are selected to be the same. The volume fraction $V_{\max }$ is 0.5 for the two cases.

Fig. 14 shows the initial configurations of the structural components for the two extension velocity cases, which include 96 and 160 structural components, respectively. According to Eq. (27), each structural component has 7 design variables. Therefore, the number of design variables for the two extension velocity cases are 672 and 1120 . Compared with the traditional density-based and level set-based topology optimization methods [33], the MMC-based method can dramatically reduce the number of design variables. This is one of the features of the MMC method. If a $400 \times 40$ mesh is used to discretize the rotating plate for the case of $v=0.5 \mathrm{~m} / \mathrm{s}$, the number of design variables in the traditional topology methods (density method and LSM) will be great than 16000 .

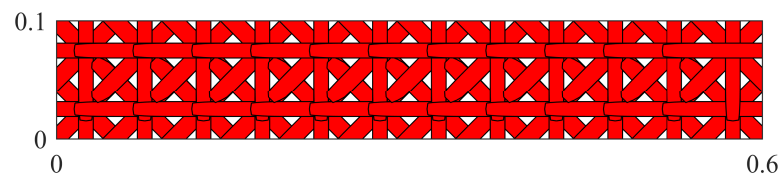

(a) $v=0.1 \mathrm{~m} / \mathrm{s}$

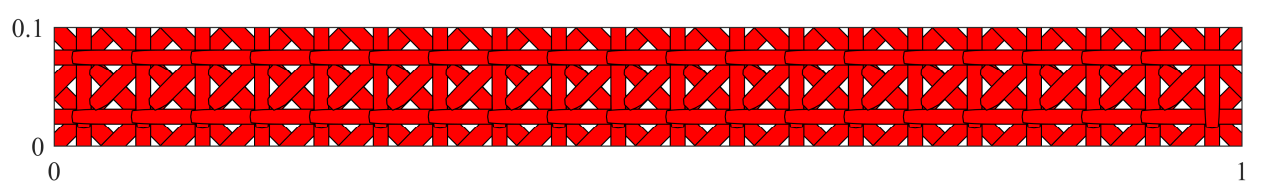

(b) $v=0.5 \mathrm{~m} / \mathrm{s}$

Fig. 14 Initial configurations of the structural components for the two extension velocity cases

The other feature of the MMC method is the controllability of the minimum thickness of the structural components during topology optimization when manufacturability or stability of a structural component is important. For the case of extension velocity $v=0.1 \mathrm{~m} / \mathrm{s}$, the topology optimization results in the first cycle of the rotating plate are depicted in Fig. 15 and Fig. 16, where the lower bounds of the thicknesses $c_{1}, c_{2}$ and $c_{3}$ of the structural components are set either $c_{\min }=0.001 \mathrm{~m}$ or $C_{\min }=0.005 \mathrm{~m}$. The comparison between the optimization results in Fig. 15(d) and Fig. 16(d) shows that the two results have similar topologies, but different thickness scales at some domains. The optimization result in Fig. 15(d) and the optimized structural components in Fig. 15(e) illustrate that without the minimum thickness control, i.e., setting $C_{\min }$ as a very small positive value, the results give very thin and unsmooth components, such as the red cycle parts in Fig. 15(d). While for the case of $c_{\min }=0.005$ 
m, Fig. 16(d) and Fig. 16(e) show that the minimum thickness control can result in wellposed optimization results.

Fig. 17 illustrates the iteration histories of the convergent objective function and the volume ratio for the cases of $c_{\min }=0.001 \mathrm{~m}$ and $c_{\min }=0.005 \mathrm{~m}$. The figure shows that the inequality volume constraints are both satisfied for the two cases. Besides, the convergence history of the objective function for the case of $c_{\min }=0.001 \mathrm{~m}$ is not that smooth with plenty of peaks during the iteration process. This is probably because that the thicknesses of some structural components are so small that the topology of the rotating plate may easily jump from one to the other when $C_{\min }=0.001 \mathrm{~m}$. Comparing the objective functions as shown in Fig. 17(a) and Fig. 17(b), it can be observed that the converged objective functions have about the same value for the two cases.

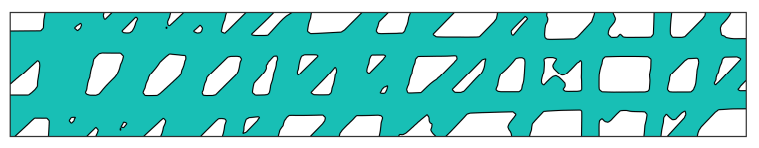

(a) Iteration No. 10

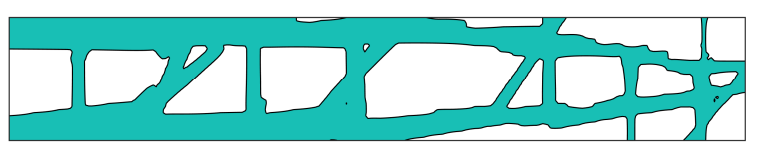

(b) Iteration No. 100

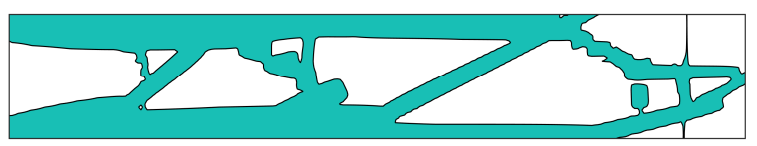

(c) Iteration No. 600

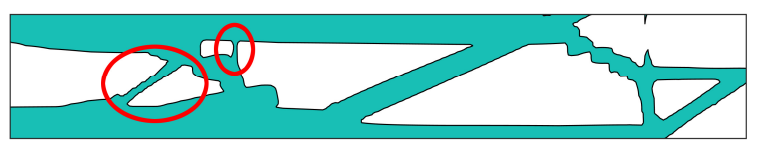

(d) Iteration No. 690

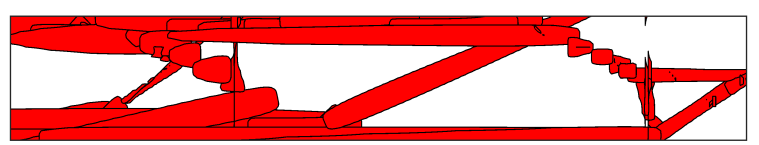

(e) Optimized components of iteration No. 690

Fig. 15 Topology optimization results for the case of $v=0.1 \mathrm{~m} / \mathrm{s}$ and $c_{\min }=0.001 \mathrm{~m}$

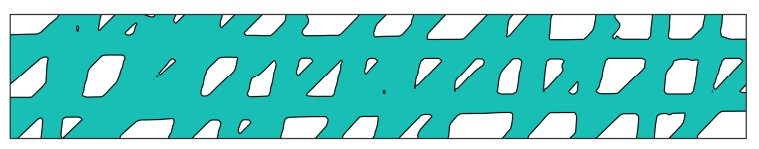

(a) Iteration No. 10

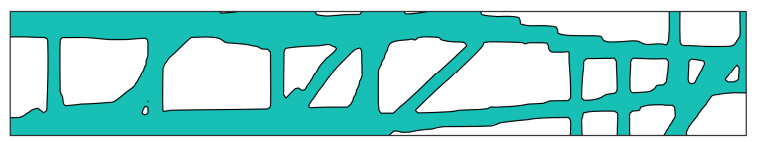

(b) Iteration No. 100 


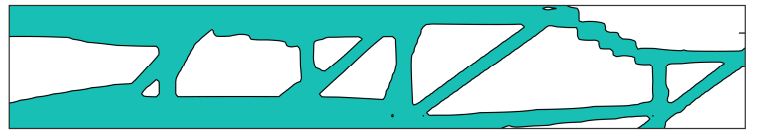

(c) Iteration No. 500

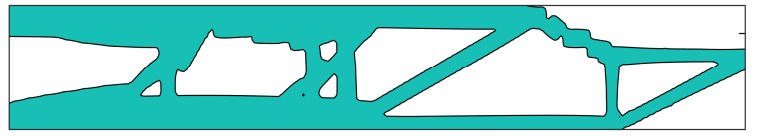

(d) Iteration No. 600

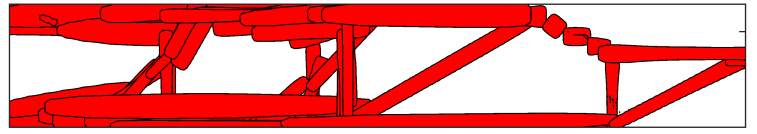

(e) Optimized components of iteration No. 600

Fig. 16 Topology optimization results for the case of $v=0.1 \mathrm{~m} / \mathrm{s}$ and $c_{\min }=0.005 \mathrm{~m}$

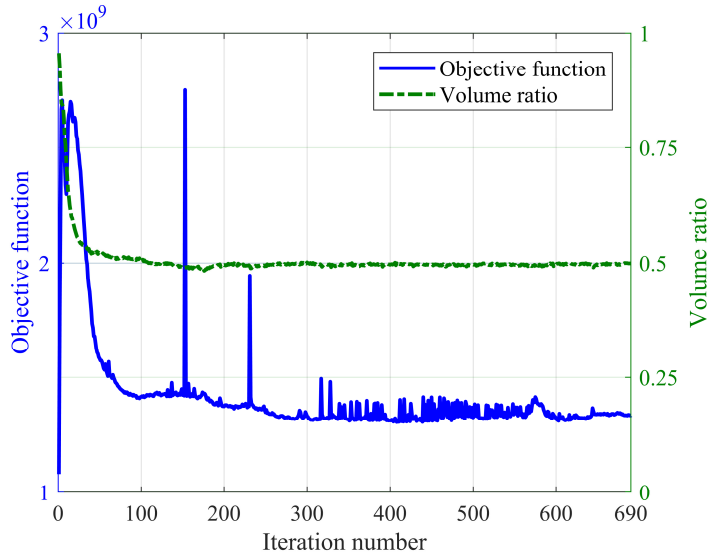

(a) $C_{\min }=0.001 \mathrm{~m}$

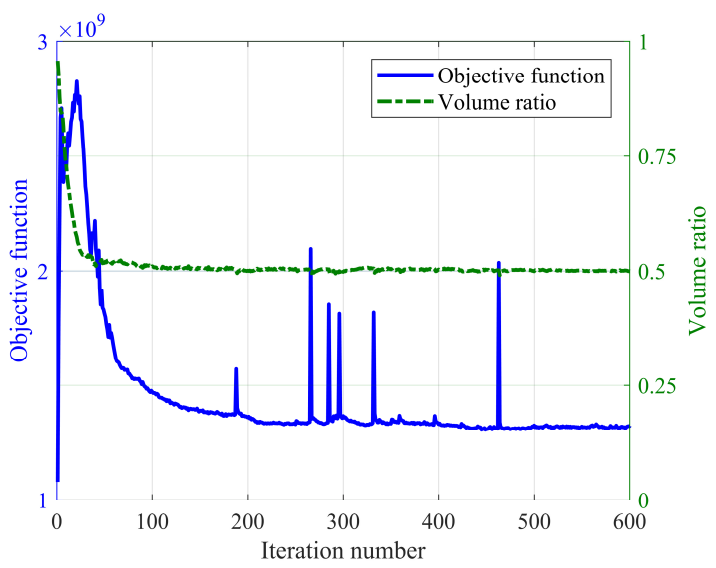

(b) $C_{\min }=0.005 \mathrm{~m}$

Fig. 17 Iteration histories of the objective function and the volume ratio for $v=0.1 \mathrm{~m} / \mathrm{s}$

Fig. 18 and Fig. 19 show the topology optimization results in the first cycle of the rotating plate in the case of extension velocity $v=0.5 \mathrm{~m} / \mathrm{s}$. The lower bounds of the thicknesses $c_{1}, c_{2}$ and $c_{3}$ are similarly set as $c_{\min }=0.001 \mathrm{~m}$ and $c_{\min }=0.005 \mathrm{~m}$ for Fig. 18 and Fig. 19, respectively. The optimized results in Fig. 18(d) and Fig. 19(d) have similar topologies, and both have more materials on the left part. Without the minimum thickness control by setting $C_{\min }$ as $0.001 \mathrm{~m}$, Fig. 18(d) depicts an unsmooth solution as some parts (see the red cycle parts) of the rotating plate have such thin structures that serious instability problem may arise. With the minimum thickness control by setting $C_{\min }$ as 0.005 m, Fig. 19(d) and Fig. 19(e) illustrate a smoother solution. Fig. 20 gives the iteration histories of the convergent objective function and the volume ratio for the cases of $c_{\min }=0.001 \mathrm{~m}$ and $c_{\min }=0.005 \mathrm{~m}$. The figure indicates that the inequality volume constraint is satisfied for the two cases. It is worth noting that the objective functions converge to almost the same value for the two cases of the minimum thickness 
control, just like that in Fig. 17. Likewise, for the case of $c_{\min }=0.001 \mathrm{~m}$, Fig. 20(a) gives an unsmooth convergence history with many peaks in the objection function.

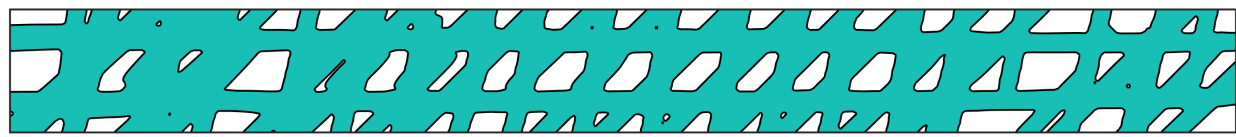

(a) Iteration No. 10

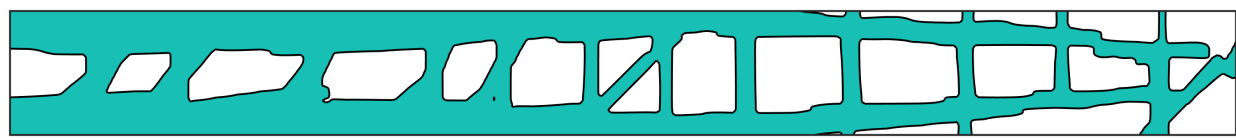

(b) Iteration No. 100

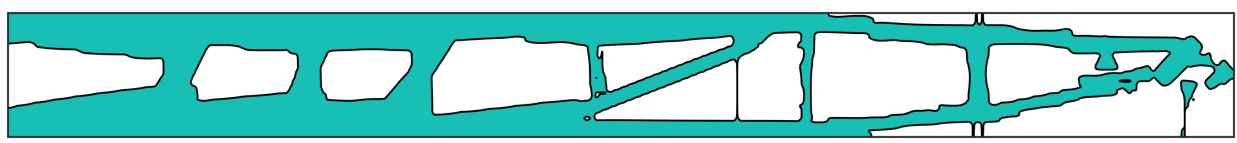

(c) Iteration No. 400

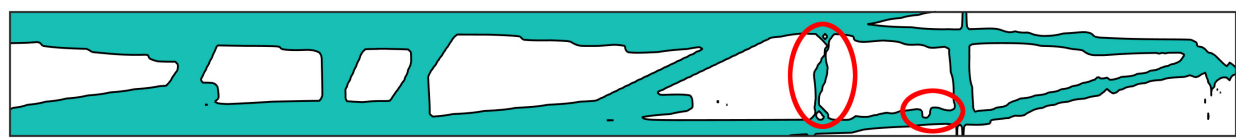

(d) Iteration No. 530

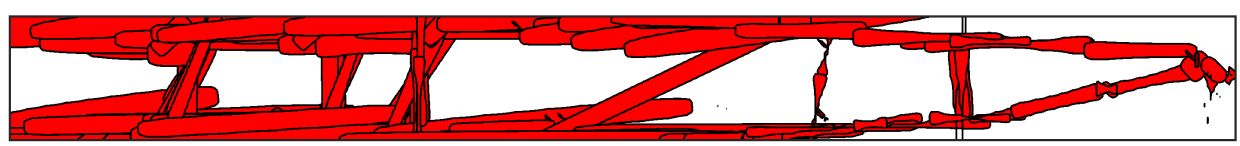

(e) Optimized components of iteration No. 530

Fig. 18 Topology optimization results for the case of $v=0.5 \mathrm{~m} / \mathrm{s}$ and $c_{\min }=0.001 \mathrm{~m}$

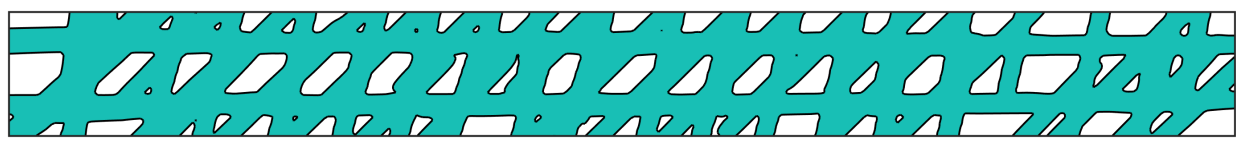

(a) Iteration No. 10

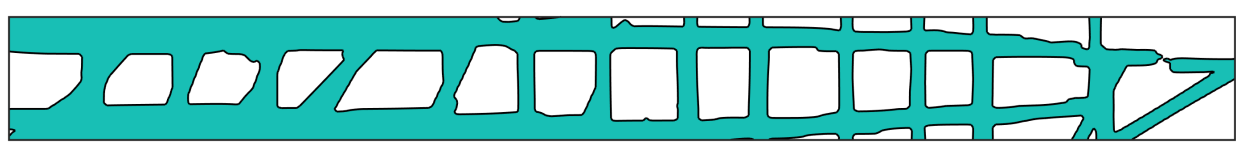

(b) Iteration No. 100

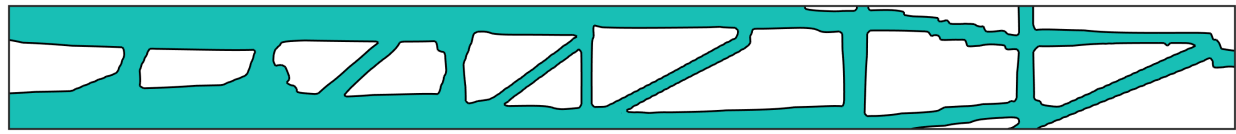

(c) Iteration No. 300

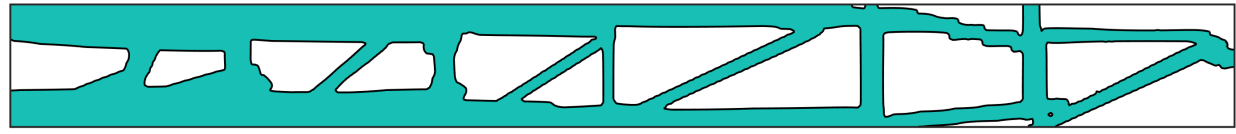

(d) Iteration No. 400

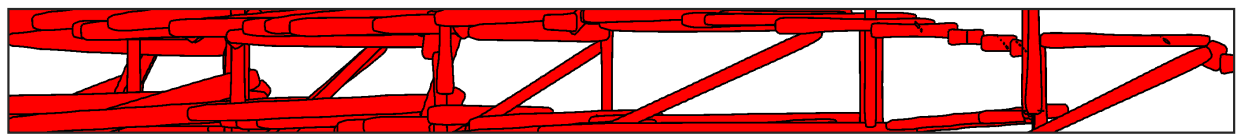

(e) Optimized components of iteration No. 400 
Fig. 19 Topology optimization results for the case of $v=0.5 \mathrm{~m} / \mathrm{s}$ and $c_{\min }=0.005 \mathrm{~m}$

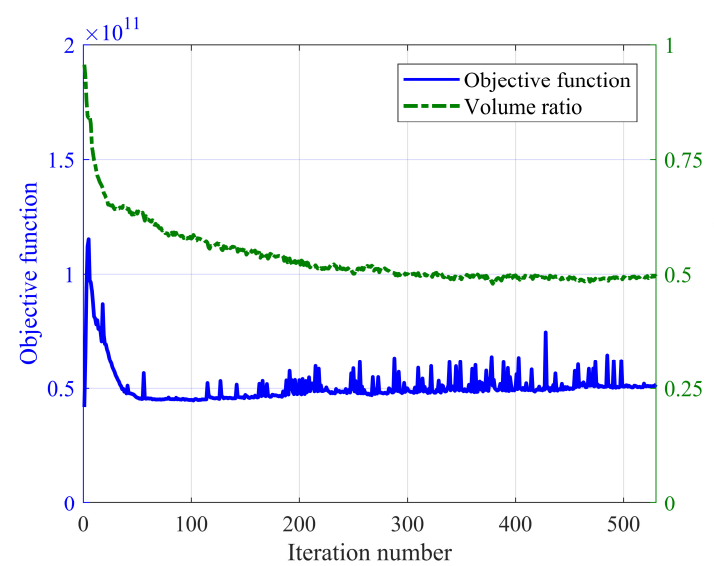

(a) $c_{\min }=0.001 \mathrm{~m}$

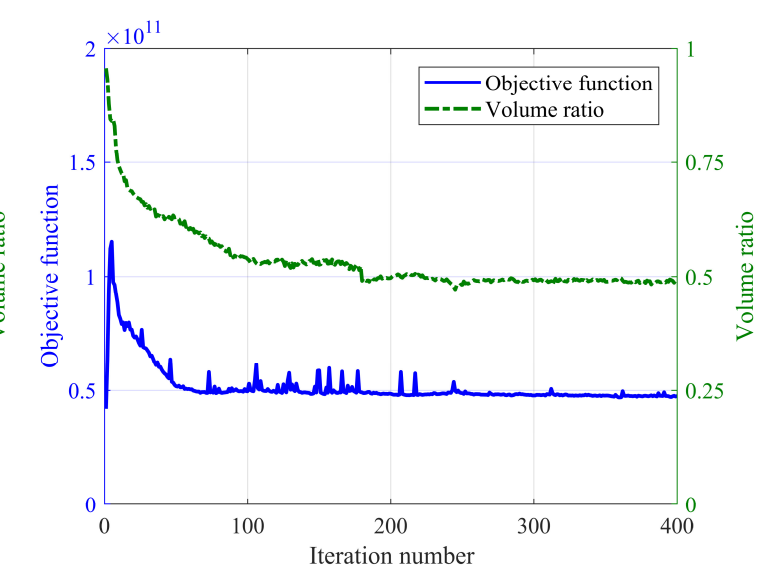

(b) $C_{\min }=0.005 \mathrm{~m}$

Fig. 20 Iteration histories of the objective function and the volume ratio for $v=0.5 \mathrm{~m} / \mathrm{s}$

The comparison between the results in Fig. 18 and Fig. 19 gives rise to the question that what is the best minimum thickness scale to control the minimum thickness of the structural components. An additional numerical example is used to explain this in Fig. 21 and Fig. 22, where $c_{\min }=0.008 \mathrm{~m}$ for the case of extension velocity $v=0.5 \mathrm{~m} / \mathrm{s}$. The optimized result in Fig. 21(d) looks like those in Fig. 18(d) and Fig. 19(d), but is a smoother and more robust one. The converged objective function for $C_{\min }=0.008 \mathrm{~m}$ case in Fig. 22 is approximately equal to those in Fig. 20. From the point of view of objective function, hence, the three optimized results in Fig. 18(d), Fig. 19(d) and Fig. 21(d) have little difference. If the minimum thickness scale of the structural component is taken into account, Fig. 19(d) and Fig. 21(d) will be superior to the one in Fig. 18(d). In practice, the best minimum thickness scale is unknown to the designers. One way to overcome this difficulty, therefore, is to try to design several times with different minimum thickness scales. The other is to include additional stability or manufacturability constraints into the optimization formulation, which will be studied in future research.

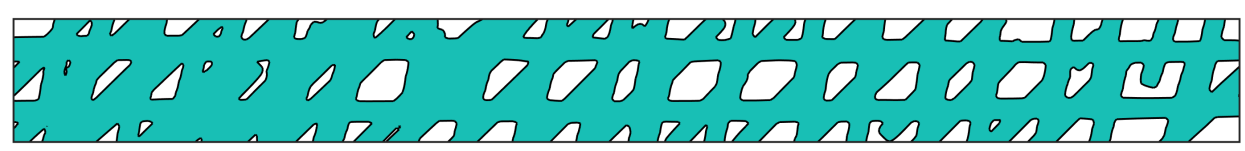

(a) Iteration No. 10

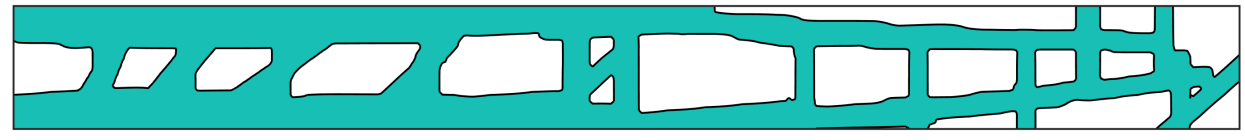

(b) Iteration No. 100 


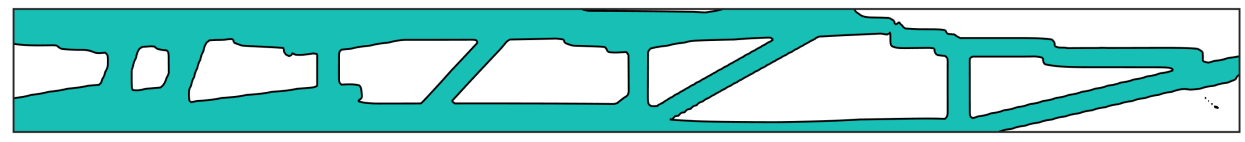

(c) Iteration No. 600

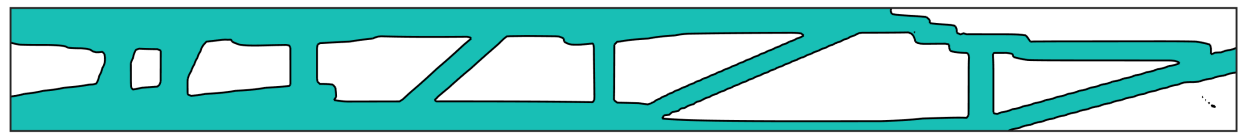

(d) Iteration No. 670

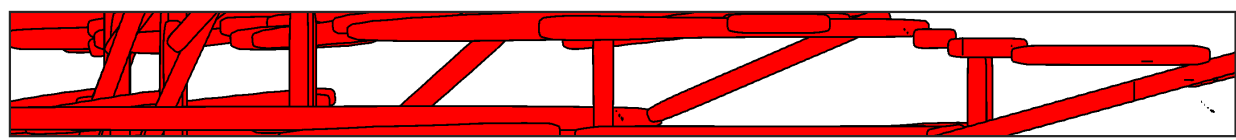

(e) Optimized components of iteration No. 670

Fig. 21 Topology optimization results for the case of $v=0.5 \mathrm{~m} / \mathrm{s}$ and $c_{\min }=0.008 \mathrm{~m}$

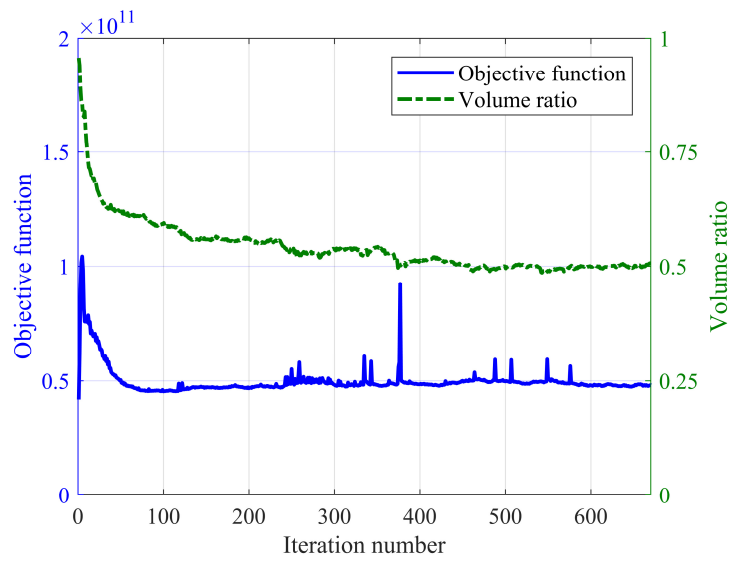

Fig. 22 Iteration histories of the objective function and the volume ratio for $v=0.5 \mathrm{~m} / \mathrm{s}$ and $c_{\min }=$

$0.008 \mathrm{~m}$

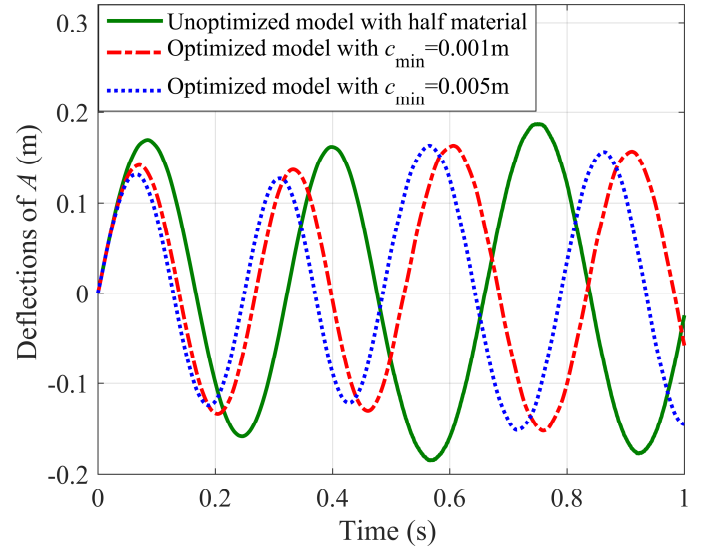

(a) $v=0.1 \mathrm{~m} / \mathrm{s}$

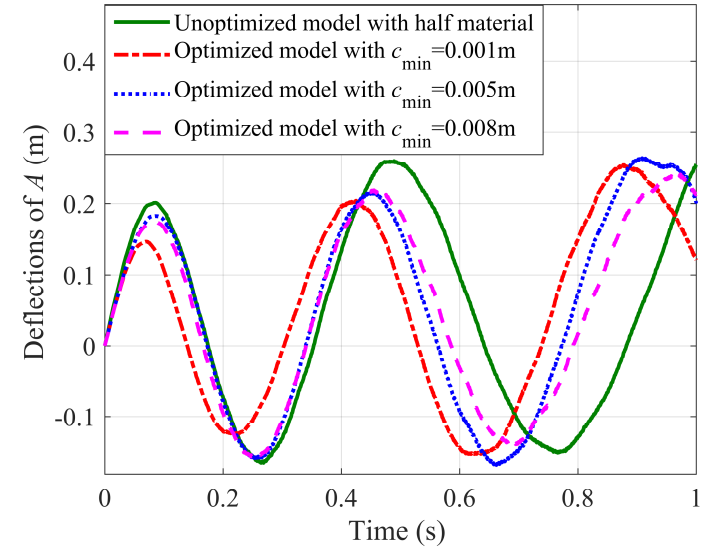

(b) $v=0.5 \mathrm{~m} / \mathrm{s}$

Fig. 23 Deflection responses of tip mass $A$ for the unoptimized and optimized models

Fig. 23 presents the deflection responses of tip mass $A$ in Fig. 13 for the unoptimized and optimized models. For a better comparison, the unoptimized model 
has only half materials, which is evenly distributed, of the initial design. It can be easily observed that the amplitudes of the optimized models have about the same values, and are all smaller than the unoptimized ones. This phenomenon indicates that the optimization of the rotating variable-length plate is successful. That is, the stiffness of the rotating plate is actually improved. Regarding the vibration frequencies, the optimized models have higher first-order vibration frequencies than the unoptimized ones.

\subsection{Topology optimization of a 3-DOF motion platform}

This example is intended to validate the proposed optimization approach to deal with a complex FMBS with many flexible variable-length bodies. As shown in Fig. 24, a 3-DOF motion platform consists of a rigid motion platform, four rigid bearings $(A, B$, $C$ and $D$ ), and two flexible variable-length connecting bars, as well as four revolute joints and two sliding joints. The rigid motion platform has a mass of $2 \mathrm{~kg}$ and a geometric size of $0.4 \mathrm{~m} \times 0.2 \mathrm{~m} \times 0.1 \mathrm{~m}$. The four rigid bearings have the same mass and geometric size, that is, $0.1 \mathrm{~kg}$ and $0.1 \mathrm{~m} \times 0.1 \mathrm{~m} \times 0.01 \mathrm{~m}$. In this study, the five rigid bodies are modeled by the natural coordinate formulation (NCF) [56]. The two variablelength connecting bars are the design domains, both of which have a width of $0.1 \mathrm{~m}$ and a thickness of $0.01 \mathrm{~m}$. The mass density of the connecting bars is $2700 \mathrm{~kg} / \mathrm{m}^{3}$, and Young's modulus and Poison's ratio are $7.2 \times 10^{9} \mathrm{~Pa}$ and 0.3 , respectively. It is assumed that the mass flows in and out at end $A$ and end $B$ of the two variable-length connecting bars.

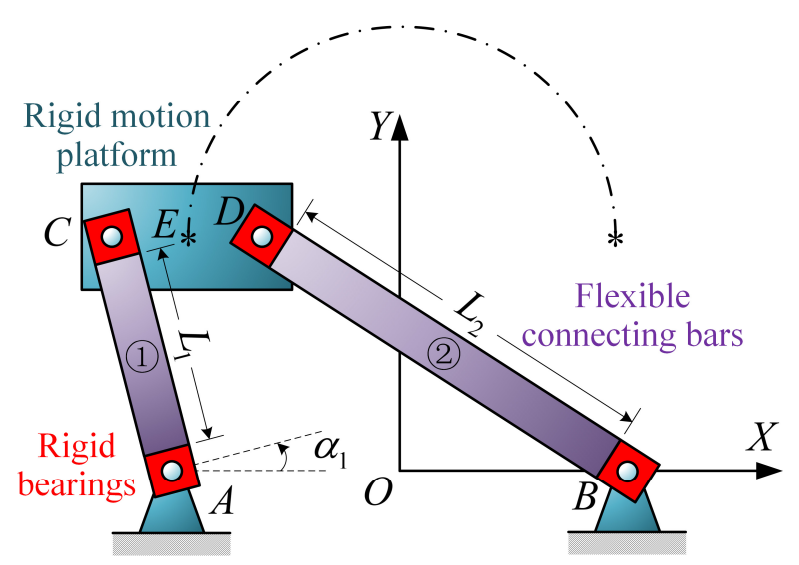

Fig. 24 The initial configuration of a 3-DOF motion platform

The rigid platform has 3 DOF of motion, which means it can freely move and rotate in the $X-Y$ plane under the three control inputs, that is, the length $L_{1}$ of 
connecting bar 1 , the length $L_{2}$ of connecting bar 2 , and the rotating angle $\alpha_{1}$ of rigid bearing $A$. In this study, to optimize the two flexible connecting bars, the motion platform is designed to translate along the dot-dashed line in Fig. 24. The trajectory of the platform centroid $E$ is

$$
\left\{\begin{array}{l}
X_{E}=0.5 \cos (\pi-\theta(t)) \\
Y_{E}=0.5 \sin (\pi-\theta(t))+0.4
\end{array},\right.
$$

with

$$
\theta(t)=-2 \pi\left(t-\frac{1}{2}\right)^{3}+\frac{3}{2} \pi t-\frac{\pi}{4} \quad t \in[0, T],
$$

where $T=1 \mathrm{~s}$ is the total analysis time. For the initial configuration of the motion platform in Fig. 24, the coordinates of the centroids of the four rigid bearings $A, B, C$ and $D$ are $(-0.4,0),(0.4,0),(-0.65,0.4)$ and $(-0.35,0.4)$, respectively. According to Eqs. (36) and (37), the desired lengths $L_{1}, L_{2}$ and the desired rotating angle $\alpha_{1}$ can be obtained as shown in Fig. 25. Fig. 25(a) indicates that the maximum length of the variable-length connecting bars is $0.87 \mathrm{~m}$. Hence, the design domains of the optimization problem are two domains of $0.87 \mathrm{~m} \times 0.1 \mathrm{~m}$. The length of the boundary element of the connecting bars can vary from $0.05 \mathrm{~m}$ to $0.1 \mathrm{~m}$. This means that when the length of the boundary element increases to $0.1 \mathrm{~m}$, two nodes of ALE-ANCF are inserted to divide the boundary element from the middle into two elements, as illustrated in Fig. 4(a). On the contrary, when the length of the boundary element reduces to $0.05 \mathrm{~m}$, two boundary elements will merge into one element, as illustrated in Fig. 4(b).

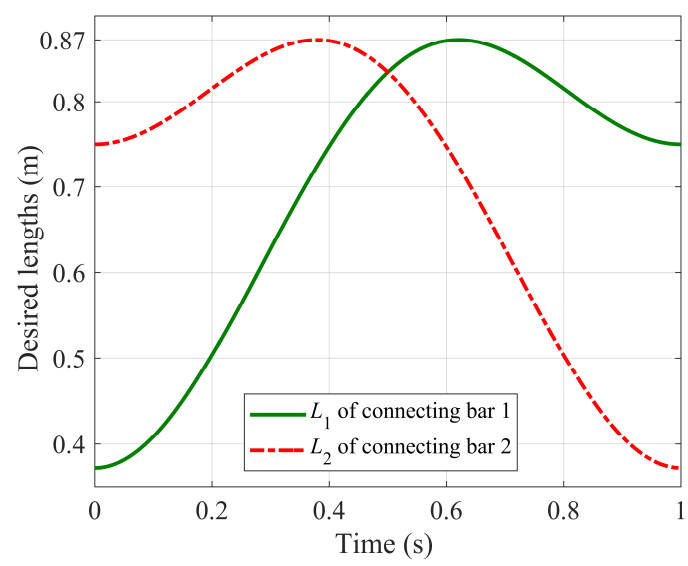

(a) Desired lengths of two connecting bars

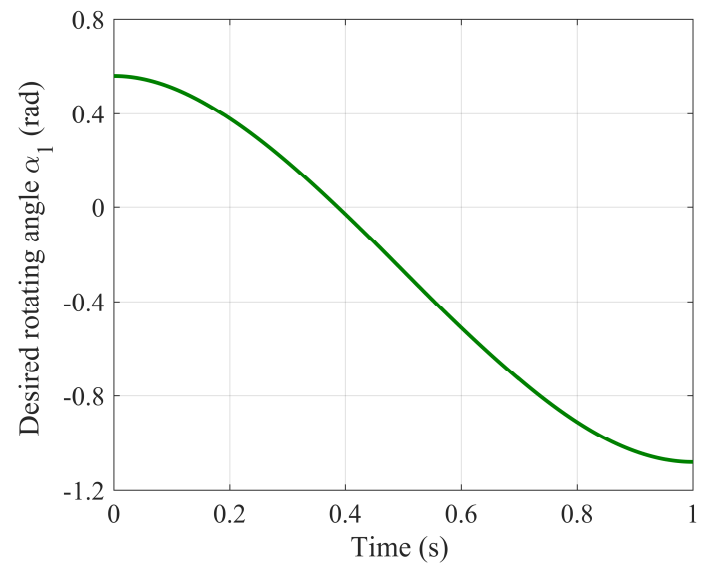

(b) Desired rotating angle of rigid bearing $A$

Fig. 25 The desired inputs for the desired movement of the platform 
To optimize the two connecting bars, the time step size is set as $0.01 \mathrm{~s}$ and the weights for the 100 time steps are all set the same. The volume fraction $V_{\max }$ of the optimization problem is set as 0.5 for the two connecting bars. Fig. 26 shows the initial configurations of the structural components for the two connecting bars, each of which has 120 components. Based on the optimization results of the rotating variable-length plate in Section 4.2, the lower bounds of the thicknesses $c_{1}, c_{2}$ and $c_{3}$ are set as $c_{\min }=$ $0.005 \mathrm{~m}$ here. The topology optimization results in the first cycle of the two connecting bars are shown in Fig. 27 and Fig. 28, where the left ends are ends $C$ and $D$ for the two connecting bars, respectively. The comparison between Fig. 27(d) and Fig. 28(d) shows that the two connecting bars have different topologies after optimization. The optimization result in Fig. 27(d) has more materials on the right part and is similar to a truss structure. While the optimization result in Fig. 28(d) is more like a tree structure with one thick tree trunk and some thin branches. This is probably due to the different driving modes for the two connecting bars. Connecting bar 1 is driven by length $L_{1}$ and rotating angle $\alpha_{1}$ at the same time, while connecting bar 2 is only driven by length $L_{2}$. As a consequence, the sets of ESL are totally different for the two connecting bars.

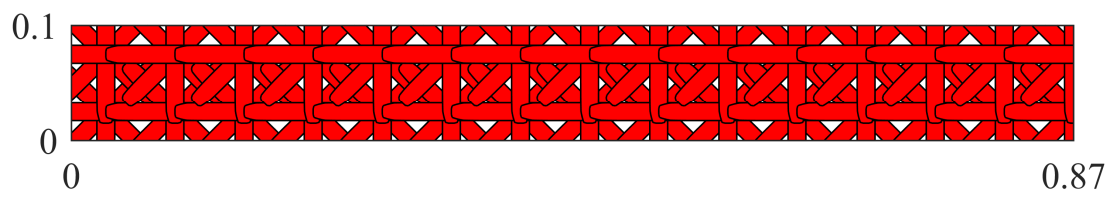

(a) Connecting bar 1

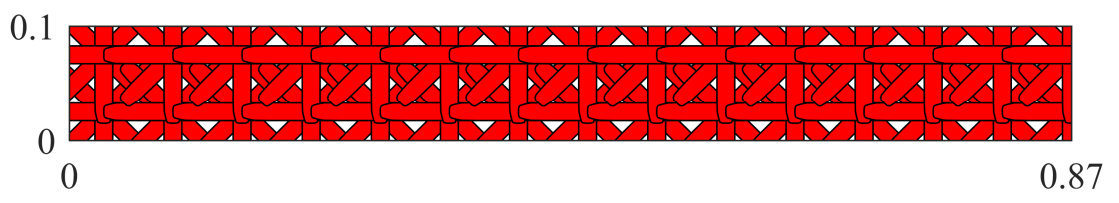

(b) Connecting bar 2

Fig. 26 Initial configurations of the structural components for the two connecting bars

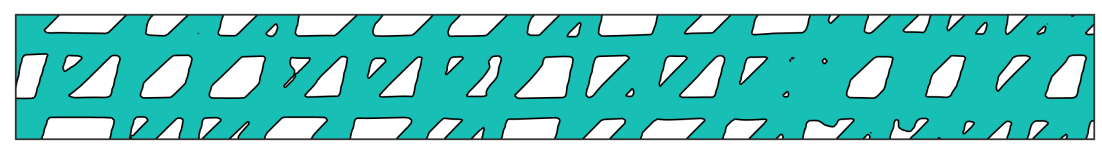

(a) Iteration No. 10

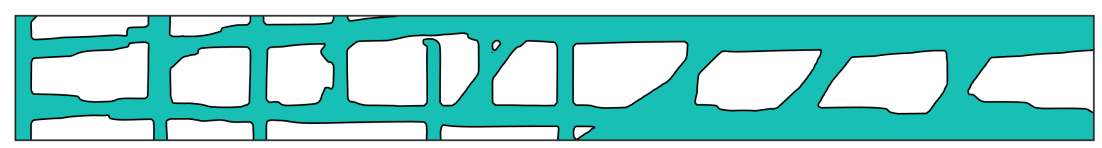

(b) Iteration No. 100 


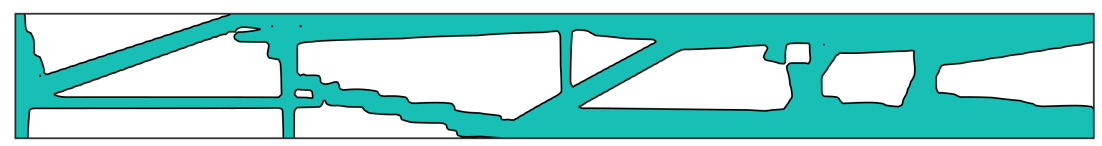

(c) Iteration No. 400

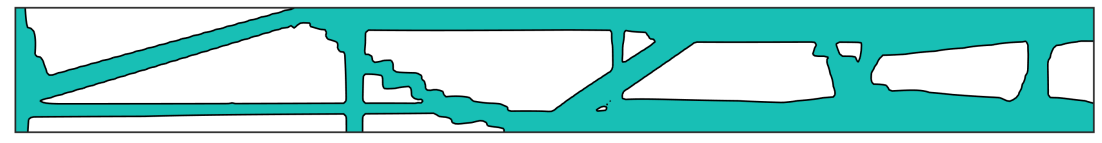

(d) Iteration No. 500

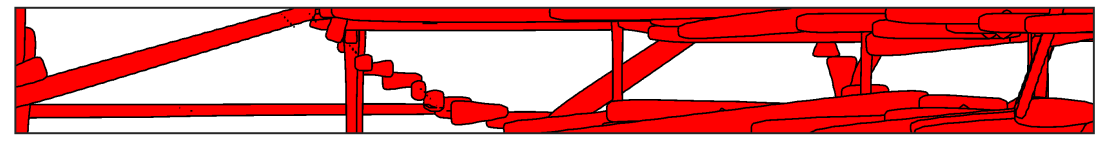

(e) Optimized components of iteration No. 500

Fig. 27 Topology optimization results for connecting bar 1

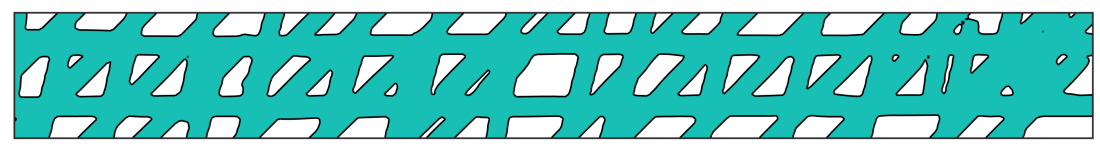

(a) Iteration No. 10

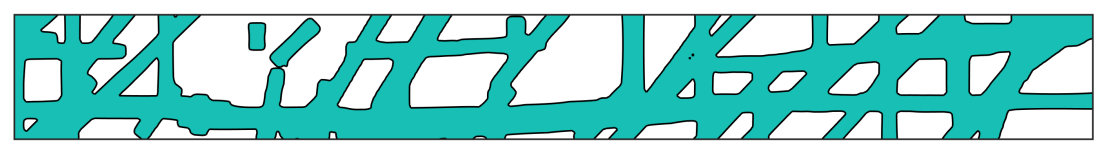

(b) Iteration No. 100

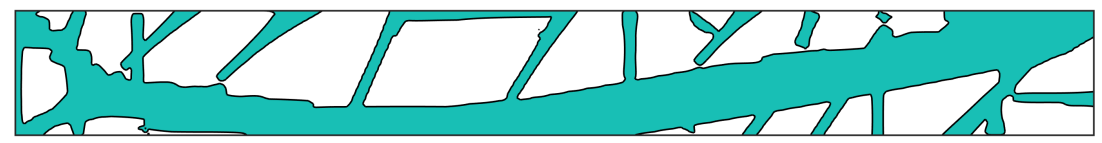

(c) Iteration No. 500

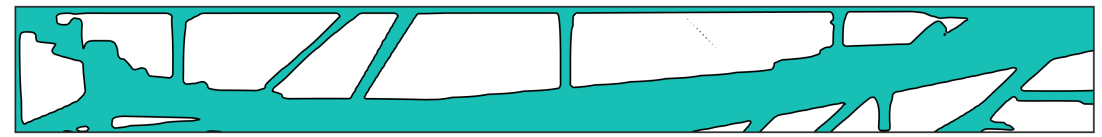

(d) Iteration No. 600

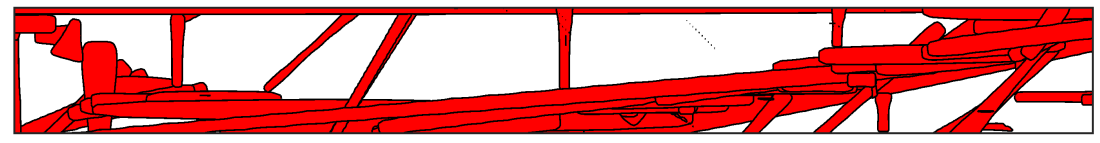

(e) Optimized components of iteration No. 600

Fig. 28 Topology optimization results for connecting bar 2

Fig. 29 gives the iteration histories of the objective function and the volume ratio for the two connecting bars. The figure indicates that the inequality volume constraint is satisfied for the two connecting bars. It is also easy to observe that the convergent objective functions have different values for the two connecting bars due to the different ESL sets for them. Fig. 30 depicts the optimized results of the 3-DOF motion platform at four moments. Fig. 31 presents the comparison of the dynamic responses of the platform $E$ between the unoptimized model and the optimized model. Likewise, the 
unoptimized model has only half materials, which is evenly distributed, of the initial design. In Fig. 31, $\Delta X_{E}, \Delta Y_{E}$ are the differences of $E$ coordinates between the actual ones and the desired ones in Eq. (36), and $\alpha_{E}$ is the rotating angle of platform E. Fig. 31 reveals that the optimized model is better than the unoptimized one as the optimized model has smaller maximum absolute deflections and rotating angles of the platform $E$ than the unoptimized model.

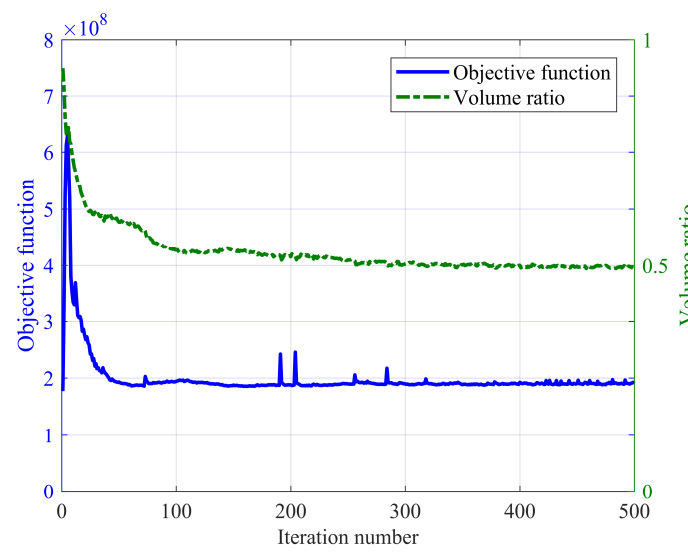

(a) Connecting bar 1

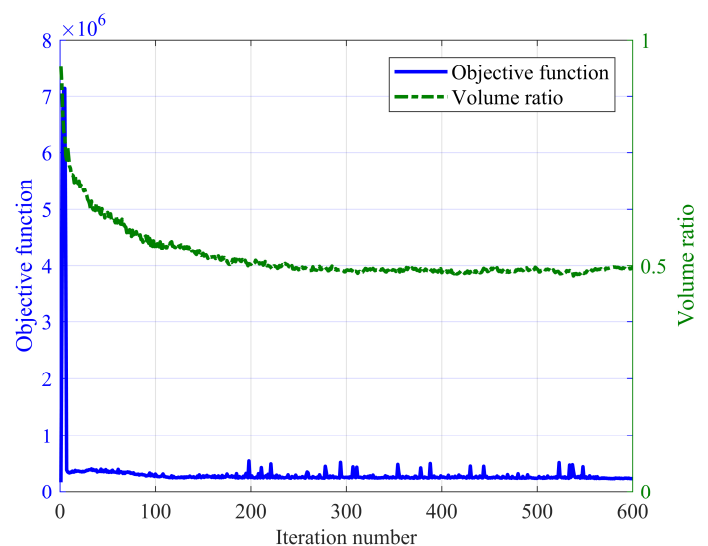

(b) Connecting bar 2

Fig. 29 Iteration histories of the objective function and the volume ratio for the two connecting bars

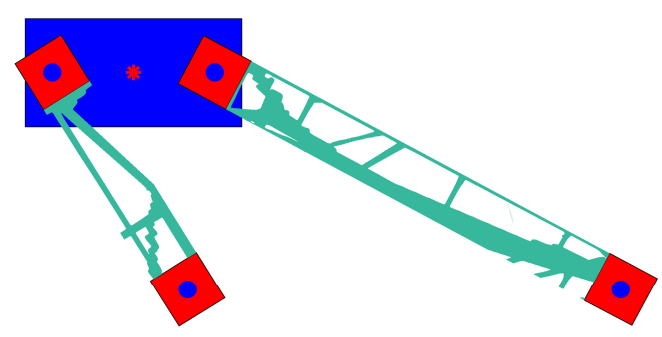

(a) $t=0 \mathrm{~s}$

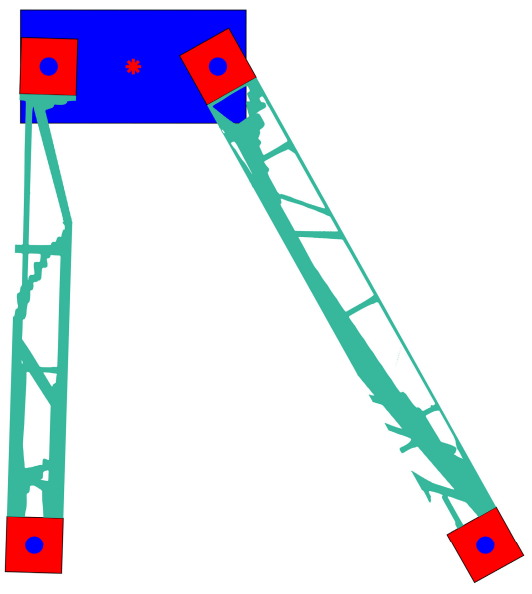

(6) $t=0.4 \mathrm{~s}$ 


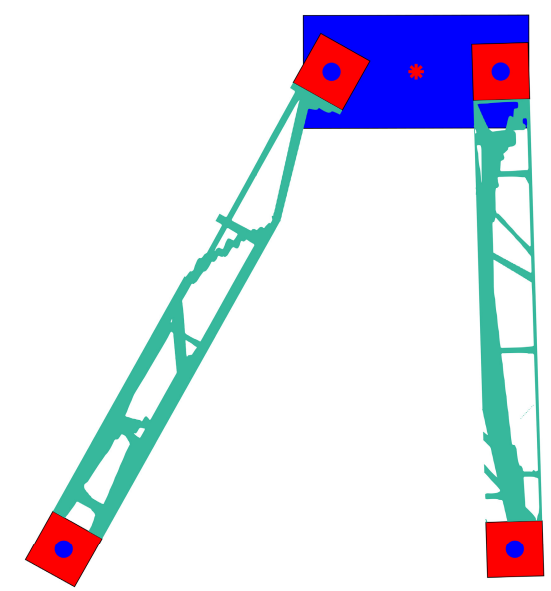

(c) $t=0.6 \mathrm{~s}$

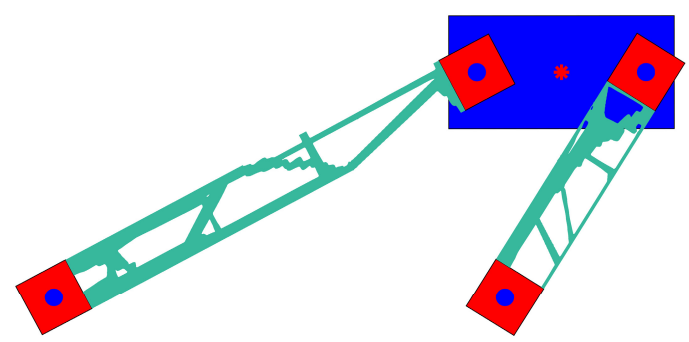

(d) $t=1 \mathrm{~s}$

Fig. 30 Optimized results of the 3-DOF motion platform at four moments

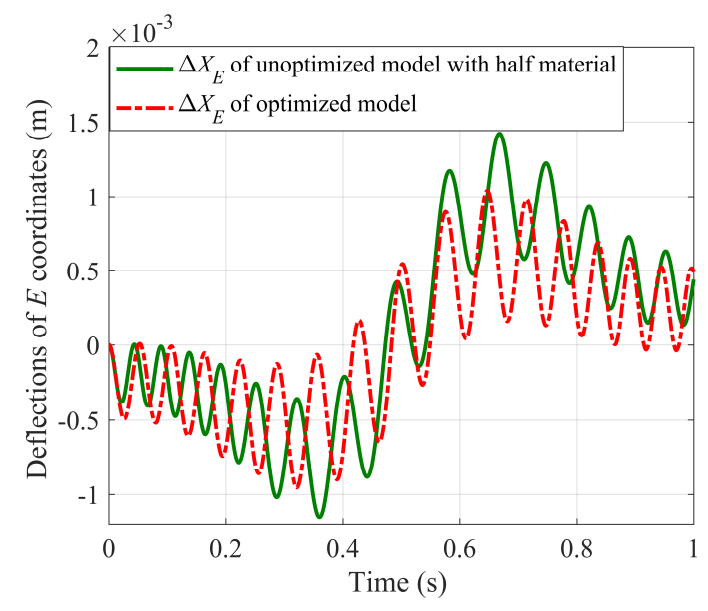

(a) Deflections of centroid $E$ in $X$ direction

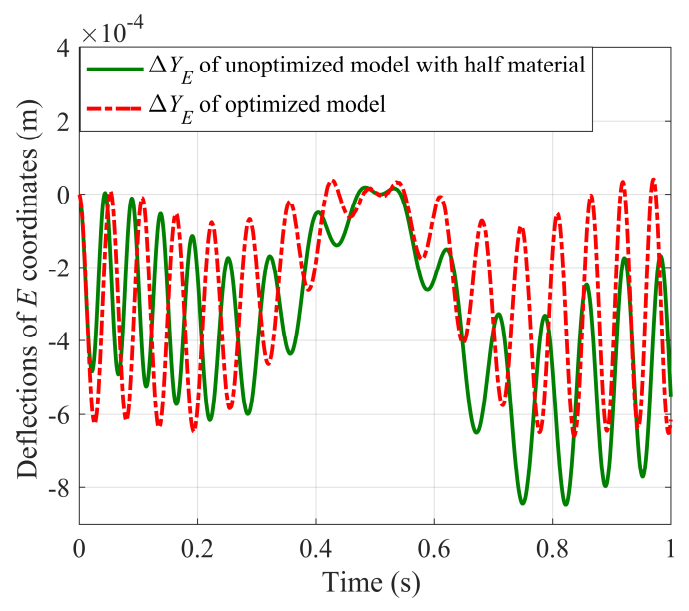

(b) Deflections of centroid $E$ in $Y$ direction

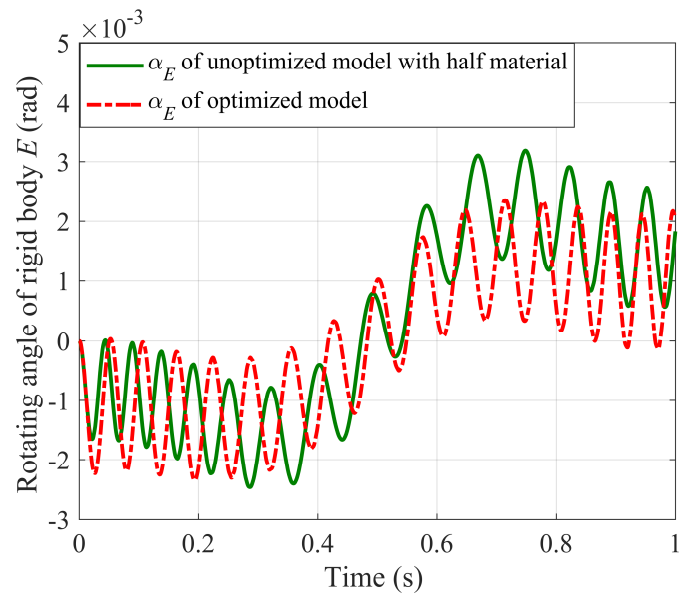

(c) Rotating angle of the platform $E$

Fig. 31 Dynamic responses of the platform $E$ for the unoptimized and optimized models 


\section{Conclusions}

The paper presents an explicit topology optimization approach for a flexible multibody system (FMBS) with variable-length undergoing both large overall motions, and large deformations by jointly using the arbitrary Lagrangian-Eulerian (ALE) description, the absolute nodal coordinate formulation (ANCF) and the moving morphable components (MMC). The ALE-ANCF modeling scheme enables one to accurately describe the dynamics of such an FMBS, and the MMC can explicitly and geometrically describe the topology of the FMBS. The paper also presents the effective computation schemes of the elastic forces and the additional inertial forces, as well as the corresponding Jacobian formulations of the thin plate element of ALE-ANCF.

The paper verifies the thin plate element of ALE-ANCF via a classic thin plate pendulum by the comparison with the conventional thin plate element of ANCF, and demonstrates the optimization approach via two planar examples. The optimization of the rotating variable-length plate under different extension velocities shows that the MMC-based topology optimization approach is able to effectively optimize an FMBS with variable-length bodies. This is due to the fact that the MMC method can dramatically reduce the number of design variables. Moreover, this example studies the minimum feature size control in the topology optimization of an FMBS by explicitly setting the lower bounds of certain geometrical design variables in the MMC method. The results reveal that the minimum thickness control is of great importance to obtain a smooth and robust result in the topology optimization of an FMBS. The best minimum thickness scale, however, is unknown to the designers, but may be found from a parameter study or by including additional stability, manufacturability or eigenfrequency constraints into the optimization formulation. The proposed optimization approach also makes it possible to optimize a complex FMBS by optimizing the two flexible connecting bars in a 3-DOF motion platform.

\section{Acknowledgments}

This work was supported in part by the National Natural Science Foundation of China under Grants 11290151 and 11472042. It was also supported in part by Postgraduate Research \& Practice Innovation Program of Jiangsu Province under Grants KYCX17_0226 and by China Scholarship Council under Grants 201706830011. 


\section{Declaration on conflict of interest}

The author(s) declared no potential conflicts of interest with respect to the research, authorship, and/or publication of this article.

\section{Appendix}

First of all, the strain tensor $\boldsymbol{\varepsilon}$ as shown in Eq. (7) can be rewritten as

$$
\boldsymbol{\varepsilon}=\left[\begin{array}{c}
\left(\mathbf{r}_{, m}^{\mathrm{T}} \mathbf{r}_{, m}-1\right) / 2 \\
\left(\mathbf{r}_{, n}^{\mathrm{T}} \mathbf{r}_{, n}-1\right) / 2 \\
\mathbf{r}_{, m}^{\mathrm{T}} \mathbf{r}_{, n}
\end{array}\right]=\left[\begin{array}{c}
\left((\mathbf{A})_{i j} q_{i} q_{j}-1\right) / 2 \\
\left((\mathbf{B})_{i j} q_{i} q_{j}-1\right) / 2 \\
(\mathbf{C})_{i j} q_{i} q_{j}
\end{array}\right],
$$

where the subscripts $i, j=1,2, \ldots, 36, \quad$ and $\quad \mathbf{A}=\mathbf{N}_{e, m}^{\mathrm{T}} \mathbf{N}_{e, m}, \quad \mathbf{B}=\mathbf{N}_{e, n}^{\mathrm{T}} \mathbf{N}_{e, n}$, $\mathbf{C}=\mathbf{N}_{e, m}^{\mathrm{T}} \mathbf{N}_{e, n}$.

Then, the invariant matrices $\left(\mathbf{K}_{1}\right)_{i k a b},\left(\mathbf{K}_{2}\right)_{i k a b},\left(\mathbf{K}_{3}\right)_{i k a b},\left(\mathbf{K}_{4}\right)_{i j k a b},\left(\mathbf{K}_{5}\right)_{i j k a b}$, $\left(\mathbf{K}_{6}\right)_{i j k a b},\left(\mathbf{G}_{1}\right)_{i k},\left(\mathbf{G}_{2}\right)_{i k},\left(\mathbf{G}_{3}\right)_{i j k}$ and $\left(\mathbf{G}_{4}\right)_{i j k}$ in Eq. (11) can be computed as follows

$$
\begin{aligned}
& \left(\mathbf{K}_{1}\right)_{i k a b}=\int_{V_{0}} \mathbf{E}_{11}^{\varepsilon}\left((\mathbf{A})_{i k}(\mathbf{A})_{a b}+(\mathbf{B})_{i k}(\mathbf{B})_{a b}\right) \mathrm{d} V, \\
& \left(\mathbf{K}_{2}\right)_{i k a b}=\int_{V_{0}} \mathbf{E}_{12}^{\varepsilon}\left((\mathbf{B})_{i k}(\mathbf{A})_{a b}+(\mathbf{A})_{i k}(\mathbf{B})_{a b}\right) \mathrm{d} V, \\
& \left(\mathbf{K}_{3}\right)_{i k a b}=\int_{V_{0}} 2 \mathbf{E}_{33}^{\varepsilon}\left((\mathbf{C})_{i k}(\mathbf{C})_{a b}+(\mathbf{C})_{k i}(\mathbf{C})_{a b}\right) \mathrm{d} V, \\
& \left(\mathbf{K}_{4}\right)_{i j k a b}=\int_{V_{0}} \mathbf{E}_{11}^{\varepsilon}\left((\mathbf{A})_{i j, q_{k}}(\mathbf{A})_{a b}+(\mathbf{B})_{i j, q_{k}}(\mathbf{B})_{a b}\right) \mathrm{d} V, \\
& \left(\mathbf{K}_{5}\right)_{i j k a b}=\int_{V_{0}} \mathbf{E}_{12}^{\varepsilon}\left((\mathbf{B})_{i j, q_{k}}(\mathbf{A})_{a b}+(\mathbf{A})_{i j, q_{k}}(\mathbf{B})_{a b}\right) \mathrm{d} V, \\
& \left(\mathbf{K}_{6}\right)_{i j k a b}=\int_{V_{0}} 4 \mathbf{E}_{33}^{\varepsilon}(\mathbf{C})_{i j, q_{k}}(\mathbf{C})_{a b} \mathrm{~d} V, \\
& \left(\mathbf{G}_{1}\right)_{i k}=\int_{V_{0}} \mathbf{E}_{11}^{\varepsilon}\left((\mathbf{A})_{i k}+(\mathbf{B})_{i k}\right) \mathrm{d} V, \\
& \left(\mathbf{G}_{2}\right)_{i k}=\int_{V_{0}} \mathbf{E}_{12}^{\varepsilon}\left((\mathbf{A})_{i k}+(\mathbf{B})_{i k}\right) \mathrm{d} V, \\
& \left(\mathbf{G}_{3}\right)_{i j k}=\int_{V_{0}} \mathbf{E}_{11}^{\varepsilon}\left((\mathbf{A})_{i j, q_{k}}+(\mathbf{B})_{i j, q_{k}}\right) \mathrm{d} V, \\
& \left(\mathbf{G}_{4}\right)_{i j k}=\int_{V_{0}} \mathbf{E}_{12}^{\varepsilon}\left((\mathbf{A})_{i j, q_{k}}+(\mathbf{B})_{i j, q_{k}}\right) \mathrm{d} V,
\end{aligned}
$$


where the subscripts $i, j, a, b=1,2, \ldots, 36$ and $\mathbf{E}_{11}^{\varepsilon}, \mathbf{E}_{12}^{\varepsilon}, \mathbf{E}_{33}^{\varepsilon}$ are the entries of the elastic coefficient matrix $\mathbf{E}^{\varepsilon}$ in Eq. (8). From Eq. (A.2), it can be observed that $\mathbf{K}_{1}$ and $\mathbf{K}_{2}$ are symmetric about $i, k$ and $a, b$, respectively. $\mathbf{K}_{4}$ and $\mathbf{K}_{5}$ are symmetric about $i, j$ and $a, b$, respectively. $\mathbf{K}_{3}, \mathbf{G}_{1}$ and $\mathbf{G}_{2}$ are symmetric about $i, k . \mathbf{K}_{6}, \mathbf{G}_{3}$ and $\mathbf{G}_{4}$ are symmetrical about $i, j$.

In Eqs. (13) and (15), the expressions of $\left(\mathbf{K}_{1}\right)_{i k a b, q_{l}},\left(\mathbf{K}_{2}\right)_{i k a b, q_{l}},\left(\mathbf{K}_{3}\right)_{i k a b, q_{l}}$, $\left(\mathbf{K}_{4}\right)_{i j k a b, q_{l}},\left(\mathbf{K}_{5}\right)_{i j k a b, q_{l}}, \quad\left(\mathbf{K}_{6}\right)_{i j k a b, q_{l}},\left(\mathbf{G}_{1}\right)_{i k, q_{l}},\left(\mathbf{G}_{2}\right)_{i k, q_{l}}, \quad\left(\mathbf{G}_{3}\right)_{i j k, q_{l}}$ and $\left(\mathbf{G}_{4}\right)_{i j k, q_{l}}$ are listed as follows

$$
\begin{aligned}
& \left(\mathbf{K}_{1}\right)_{i k a b, q_{l}}=\int_{V_{0}} \mathbf{E}_{11}^{\varepsilon}\left((\mathbf{A})_{i k, q_{l}}(\mathbf{A})_{a b}+(\mathbf{A})_{i k}(\mathbf{A})_{a b, q_{l}}\right) \mathrm{d} V \\
& +\int_{V_{0}} \mathbf{E}_{11}^{\varepsilon}\left((\mathbf{B})_{i k, q_{l}}(\mathbf{B})_{a b}+(\mathbf{B})_{i k}(\mathbf{B})_{a b, q_{l}}\right) \mathrm{d} V, \\
& \left(\mathbf{K}_{2}\right)_{i k a b, q_{l}}=\int_{V_{0}} \mathbf{E}_{12}^{\varepsilon}\left((\mathbf{B})_{i k, q_{l}}(\mathbf{A})_{a b}+(\mathbf{B})_{i k}(\mathbf{A})_{a b, q_{l}}\right) \mathrm{d} V \\
& +\int_{V_{0}} \mathbf{E}_{12}^{\varepsilon}\left((\mathbf{A})_{i k, q_{l}}(\mathbf{B})_{a b}+(\mathbf{A})_{i k}(\mathbf{B})_{a b, q_{l}}\right) \mathrm{d} V, \\
& \left(\mathbf{K}_{3}\right)_{i k a b, q_{l}}=\int_{V_{0}} 2 \mathbf{E}_{33}^{\varepsilon}\left((\mathbf{C})_{i k, q_{l}}(\mathbf{C})_{a b}+(\mathbf{C})_{i k}(\mathbf{C})_{a b, q_{l}}\right) \mathrm{d} V \\
& +\int_{V_{0}} 2 \mathbf{E}_{33}^{\varepsilon}\left((\mathbf{C})_{k i, q_{l}}(\mathbf{C})_{a b}+(\mathbf{C})_{k i}(\mathbf{C})_{a b, q_{l}}\right) \mathrm{d} V, \\
& \left(\mathbf{K}_{4}\right)_{i j k a b, q_{l}}=\int_{V_{0}} \mathbf{E}_{11}^{\varepsilon}\left((\mathbf{A})_{i j, q_{k} q_{l}}(\mathbf{A})_{a b}+(\mathbf{A})_{i j, q_{k}}(\mathbf{A})_{a b, q_{l}}\right) \mathrm{d} V \\
& +\int_{V_{0}} \mathbf{E}_{11}^{\varepsilon}\left((\mathbf{B})_{i j, q_{k} q_{l}}(\mathbf{B})_{a b}+(\mathbf{B})_{i j, q_{k}}(\mathbf{B})_{a b, q_{l}}\right) \mathrm{d} V, \\
& \left(\mathbf{K}_{5}\right)_{i j k a b, q_{l}}=\int_{V_{0}} \mathbf{E}_{12}^{\varepsilon}\left((\mathbf{B})_{i j, q_{k} q_{l}}(\mathbf{A})_{a b}+(\mathbf{B})_{i j, q_{k}}(\mathbf{A})_{a b, q_{l}}\right) \mathrm{d} V \\
& +\int_{V_{0}} \mathbf{E}_{12}^{\varepsilon}\left((\mathbf{A})_{i j, q_{k} q_{l}}(\mathbf{B})_{a b}+(\mathbf{A})_{i j, q_{k}}(\mathbf{B})_{a b, q_{l}}\right) \mathrm{d} V, \\
& \left(\mathbf{K}_{6}\right)_{i j k a b, q_{l}}=\int_{V_{0}} 4 \mathbf{E}_{33}^{\varepsilon}\left((\mathbf{C})_{i j, q_{k} q_{l}}(\mathbf{C})_{a b}+(\mathbf{C})_{i j, q_{k}}(\mathbf{C})_{a b, q_{l}}\right) \mathrm{d} V \text {, } \\
& \left(\mathbf{G}_{1}\right)_{i k, q_{l}}=\int_{V_{0}} \mathbf{E}_{11}^{\varepsilon}\left((\mathbf{A})_{i k, q_{l}}+(\mathbf{B})_{i k, q_{l}}\right) \mathrm{d} V, \\
& \left(\mathbf{G}_{2}\right)_{i k, q_{l}}=\int_{V_{0}} \mathbf{E}_{12}^{\varepsilon}\left((\mathbf{A})_{i k, q_{l}}+(\mathbf{B})_{i k, q_{l}}\right) \mathrm{d} V \\
& \left(\mathbf{G}_{3}\right)_{i j k, q_{l}}=\int_{V_{0}} \mathbf{E}_{11}^{\varepsilon}\left((\mathbf{A})_{i j, q_{k} q_{l}}+(\mathbf{B})_{i j, q_{k} q_{l}}\right) \mathrm{d} V, \\
& \left(\mathbf{G}_{4}\right)_{i j k, q_{l}}=\int_{V_{0}} \mathbf{E}_{12}^{\varepsilon}\left((\mathbf{A})_{i j, q_{k} q_{l}}+(\mathbf{B})_{i j, q_{k} q_{l}}\right) \mathrm{d} V
\end{aligned}
$$

where the subscripts $i, j, a, b=1,2, \ldots, 36$. 
Besides, the expressions of $\left(\mathbf{H}_{1}\right)_{k a},\left(\mathbf{H}_{2}\right)_{k a}, \ldots,\left(\mathbf{H}_{14}\right)_{k a}$ and $\left(\mathbf{H}_{15}\right)_{k b a}$, $\left(\mathbf{H}_{16}\right)_{k b a}, \ldots,\left(\mathbf{H}_{28}\right)_{k b a}$ in Eqs. (17) and (18) are listed as follows

$$
\begin{aligned}
& \left(\mathbf{H}_{1}\right)_{k a}=2 \rho \int_{V_{0}}\left(\mathbf{N}_{e}\right)_{j k}\left(\mathbf{N}_{e, m_{1}}\right)_{j a} \mathrm{~d} V, \\
& \left(\mathbf{H}_{2}\right)_{k a}=2 \rho \int_{V_{0}}\left(\mathbf{N}_{e}\right)_{j k}\left(\mathbf{N}_{e, n_{1}}\right)_{j a} \mathrm{~d} V, \\
& \left(\mathbf{H}_{3}\right)_{k a}=2 \rho \int_{V_{0}}\left(\mathbf{N}_{e}\right)_{j k}\left(\mathbf{N}_{e, m_{2}}\right)_{j a} \mathrm{~d} V, \\
& \left(\mathbf{H}_{4}\right)_{k a}=2 \rho \int_{V_{0}}\left(\mathbf{N}_{e}\right)_{j k}\left(\mathbf{N}_{e, n_{2}}\right)_{j a} \mathrm{~d} V, \\
& \left(\mathbf{H}_{5}\right)_{k a}=\rho \int_{V_{0}}\left(\mathbf{N}_{e}\right)_{j k}\left(\mathbf{N}_{e, m_{1} m_{1}}\right)_{j a} \mathrm{~d} V, \\
& \left(\mathbf{H}_{6}\right)_{k a}=\rho \int_{V_{0}}\left(\mathbf{N}_{e}\right)_{j k}\left(\mathbf{N}_{e, n_{1} n_{1}}\right)_{j a} \mathrm{~d} V, \\
& \left(\mathbf{H}_{7}\right)_{k a}=\rho \int_{V_{0}}\left(\mathbf{N}_{e}\right)_{j k}\left(\mathbf{N}_{e, m_{2} m_{2}}\right)_{j a} \mathrm{~d} V, \\
& \left(\mathbf{H}_{8}\right)_{k a}=\rho \int_{V_{0}}\left(\mathbf{N}_{e}\right)_{j k}\left(\mathbf{N}_{e, n_{2} n_{2}}\right)_{j a} \mathrm{~d} V,=2 \rho \int_{V_{0}}\left(\mathbf{N}_{e}\right)_{j k}\left(\mathbf{N}_{e, m_{1} n_{1}}\right)_{j a} \mathrm{~d} V, \\
& \left(\mathbf{H}_{10}\right)_{k a}=2 \rho \int_{V_{0}}\left(\mathbf{N}_{e}\right)_{j k}\left(\mathbf{N}_{e, m_{1} m_{2}}\right)_{j a} \mathrm{~d} V, \\
& \left(\mathbf{H}_{11}\right)_{k a}=2 \rho \int_{V_{0}}\left(\mathbf{N}_{e}\right)_{j k}\left(\mathbf{N}_{e, m_{1} n_{2}}\right)_{j a} \mathrm{~d} V, \\
& \left(\mathbf{H}_{12}\right)_{k a}=2 \rho \int_{V_{0}}\left(\mathbf{N}_{e}\right)_{j k}\left(\mathbf{N}_{e, n_{1} m_{2}}\right)_{j a} \mathrm{~d} V, \\
& \left.\left(\mathbf{H}_{13}\right)_{k a}=2 \rho \int_{V_{0}}\left(\mathbf{N}_{e}\right)_{j k}\left(\mathbf{N}_{e, n_{1} n_{2}}\right)_{j a} \mathrm{~d} V,\right)_{k a}=2 \rho \int_{V_{0}}\left(\mathbf{N}_{e}\right)_{j k}\left(\mathbf{N}_{e, m_{2} n_{2}}\right)_{j a} \mathrm{~d} V,
\end{aligned}
$$

and

$$
\begin{aligned}
& \left(\mathbf{H}_{15}\right)_{k b a}=2 \rho \int_{V_{0}}\left(\mathbf{N}_{e, q_{k}}\right)_{j b}\left(\mathbf{N}_{e, m_{1}}\right)_{j a} \mathrm{~d} V, \\
& \left(\mathbf{H}_{16}\right)_{k b a}=2 \rho \int_{V_{0}}\left(\mathbf{N}_{e, q_{k}}\right)_{j b}\left(\mathbf{N}_{e, n_{1}}\right)_{j a} \mathrm{~d} V, \\
& \left(\mathbf{H}_{17}\right)_{k b a}=2 \rho \int_{V_{0}}\left(\mathbf{N}_{e, q_{k}}\right)_{j b}\left(\mathbf{N}_{e, m_{2}}\right)_{j a} \mathrm{~d} V, \\
& \left(\mathbf{H}_{18}\right)_{k b a}=2 \rho \int_{V_{0}}\left(\mathbf{N}_{e, q_{k}}\right)_{j b}\left(\mathbf{N}_{e, n_{2}}\right)_{j a} \mathrm{~d} V, \\
& \left(\mathbf{H}_{19}\right)_{k b a}=\rho \int_{V_{0}}\left(\mathbf{N}_{e, q_{k}}\right)_{j b}\left(\mathbf{N}_{e, m_{1} m_{1}}\right)_{j a} \mathrm{~d} V,
\end{aligned}
$$




$$
\begin{aligned}
& \left(\mathbf{H}_{20}\right)_{k b a}=\rho \int_{V_{0}}\left(\mathbf{N}_{e, q_{k}}\right)_{j b}\left(\mathbf{N}_{e, n_{1} n_{1}}\right)_{j a} \mathrm{~d} V, \\
& \left(\mathbf{H}_{21}\right)_{k b a}=\rho \int_{V_{0}}\left(\mathbf{N}_{e, q_{k}}\right)_{j b}\left(\mathbf{N}_{e, m_{2} m_{2}}\right)_{j a} \mathrm{~d} V, \\
& \left(\mathbf{H}_{22}\right)_{k b a}=\rho \int_{V_{0}}\left(\mathbf{N}_{e, q_{k}}\right)_{j b}\left(\mathbf{N}_{e, n_{2} n_{2}}\right)_{j a} \mathrm{~d} V, \\
& \left(\mathbf{H}_{23}\right)_{k b a}=2 \rho \int_{V_{0}}\left(\mathbf{N}_{e, q_{k}}\right)_{j b}\left(\mathbf{N}_{e, m_{1} n_{1}}\right)_{j a} \mathrm{~d} V, \\
& \left(\mathbf{H}_{24}\right)_{k b a}=2 \rho \int_{V_{0}}\left(\mathbf{N}_{e, q_{k}}\right)_{j b}\left(\mathbf{N}_{e, m_{1} m_{2}}\right)_{j a} \mathrm{~d} V, \\
& \left(\mathbf{H}_{25}\right)_{k b a}=2 \rho \int_{V_{0}}\left(\mathbf{N}_{e, q_{k}}\right)_{j b}\left(\mathbf{N}_{e, m_{1} n_{2}}\right)_{j a} \mathrm{~d} V, \\
& \left(\mathbf{H}_{26}\right)_{k b a}=2 \rho \int_{V_{0}}\left(\mathbf{N}_{e, q_{k}}\right)_{j b}\left(\mathbf{N}_{e, n_{1} m_{2}}\right)_{j a} \mathrm{~d} V, \\
& \left(\mathbf{H}_{27}\right)_{k b a}=2 \rho \int_{V_{0}}\left(\mathbf{N}_{e, q_{k}}\right)_{j b}\left(\mathbf{N}_{e, n_{1} n_{2}}\right)_{j a} \mathrm{~d} V, \\
& \left(\mathbf{H}_{28}\right)_{k b a}=2 \rho \int_{V_{0}}\left(\mathbf{N}_{e, q_{k}}\right)_{j b}\left(\mathbf{N}_{e, m_{2} n_{2}}\right)_{j a} \mathrm{~d} V .
\end{aligned}
$$

In Eqs. (A.4) and (A.5), the subscripts $a, b=1,2, \ldots, 36$ and $j=1,2,3$.

Likewise, the expressions of $\left(\mathbf{H}_{1}\right)_{k a, q_{l}},\left(\mathbf{H}_{2}\right)_{k a, q_{l}}, \ldots,\left(\mathbf{H}_{14}\right)_{k a, q_{l}}$ and $\left(\mathbf{H}_{15}\right)_{k b a, q_{l}}$, $\left(\mathbf{H}_{16}\right)_{k b a, q_{l}}, \ldots,\left(\mathbf{H}_{28}\right)_{k b a, q_{l}}$ in Eqs. (19)-(22) are listed as follows

$$
\begin{aligned}
& \left(\mathbf{H}_{1}\right)_{k a, q_{l}}=2 \rho \int_{V_{0}}\left[\left(\mathbf{N}_{e, q_{l}}\right)_{j k}\left(\mathbf{N}_{e, m_{1}}\right)_{j a}+\left(\mathbf{N}_{e}\right)_{j k}\left(\mathbf{N}_{e, m_{1} q_{l}}\right)_{j a}\right] \mathrm{d} V, \\
& \left(\mathbf{H}_{2}\right)_{k a, q_{l}}=2 \rho \int_{V_{0}}\left[\left(\mathbf{N}_{e, q_{l}}\right)_{j k}\left(\mathbf{N}_{e, n_{1}}\right)_{j a}+\left(\mathbf{N}_{e}\right)_{j k}\left(\mathbf{N}_{e, n_{1} q_{l}}\right)_{j a}\right] \mathrm{d} V, \\
& \left(\mathbf{H}_{3}\right)_{k a, q_{l}}=2 \rho \int_{V_{0}}\left[\left(\mathbf{N}_{e, q_{l}}\right)_{j k}\left(\mathbf{N}_{e, m_{2}}\right)_{j a}+\left(\mathbf{N}_{e}\right)_{j k}\left(\mathbf{N}_{e, m_{2} q_{l}}\right)_{j a}\right] \mathrm{d} V, \\
& \left(\mathbf{H}_{4}\right)_{k a, q_{l}}=2 \rho \int_{V_{0}}\left[\left(\mathbf{N}_{e, q_{l}}\right)_{j k}\left(\mathbf{N}_{e, n_{2}}\right)_{j a}+\left(\mathbf{N}_{e}\right)_{j k}\left(\mathbf{N}_{e, n_{2} q_{l}}\right)_{j a}\right] \mathrm{d} V, \\
& \left(\mathbf{H}_{5}\right)_{k a, q_{l}}=\rho \int_{V_{0}}\left[\left(\mathbf{N}_{e, q_{l} l}\right)_{j k}\left(\mathbf{N}_{e, m_{1} m_{1}}\right)_{j a}+\left(\mathbf{N}_{e}\right)_{j k}\left(\mathbf{N}_{e, m_{1} m_{1} q_{l}}\right)_{j a}\right] \mathrm{d} V, \\
& \left(\mathbf{H}_{6}\right)_{k a, q_{l}}=\rho \int_{V_{0}}\left[\left(\mathbf{N}_{e, q_{l}}\right)_{j k}\left(\mathbf{N}_{e, n_{1} n_{1}}\right)_{j a}+\left(\mathbf{N}_{e}\right)_{j k}\left(\mathbf{N}_{e, n_{1} n_{1} q_{l}}\right)_{j a}\right] \mathrm{d} V, \\
& \left(\mathbf{H}_{7}\right)_{k a, q_{l}}=\rho \int_{V_{0}}\left[\left(\mathbf{N}_{e, q_{l} l}\right)_{j k}\left(\mathbf{N}_{e, m_{2} m_{2}}\right)_{j a}+\left(\mathbf{N}_{e}\right)_{j k}\left(\mathbf{N}_{e, m_{2} m_{2} q_{l}}\right)_{j a}\right] \mathrm{d} V, \\
& \left(\mathbf{H}_{8}\right)_{k a, q_{l}}=\rho \int_{V_{0}}\left[\left(\mathbf{N}_{e, q_{l} l}\right)_{j k}\left(\mathbf{N}_{e, n_{2} n_{2}}\right)_{j a}+\left(\mathbf{N}_{e}\right)_{j k}\left(\mathbf{N}_{e, n_{2} n_{2} q_{l}}\right)_{j a}\right] \mathrm{d} V, \\
& \left(\mathbf{H}_{9}\right)_{k a, q_{l}}=2 \rho \int_{V_{0}}\left[\left(\mathbf{N}_{e, q_{l}}\right)_{j k}\left(\mathbf{N}_{e, m_{1} n_{1}}\right)_{j a}+\left(\mathbf{N}_{e}\right)_{j k}\left(\mathbf{N}_{e, m_{1} n_{1} q_{l}}\right)_{j a}\right] \mathrm{d} V,
\end{aligned}
$$




$$
\begin{aligned}
& \left(\mathbf{H}_{10}\right)_{k a, q_{l}}=2 \rho \int_{V_{0}}\left[\left(\mathbf{N}_{e, q_{l}}\right)_{j k}\left(\mathbf{N}_{e, m_{1} m_{2}}\right)_{j a}+\left(\mathbf{N}_{e}\right)_{j k}\left(\mathbf{N}_{e, m_{1} m_{2} q_{l}}\right)_{j a}\right] \mathrm{d} V, \\
& \left(\mathbf{H}_{11}\right)_{k a, q_{l}}=2 \rho \int_{V_{0}}\left[\left(\mathbf{N}_{e, q_{l}}\right)_{j k}\left(\mathbf{N}_{e, m_{1} n_{2}}\right)_{j a}+\left(\mathbf{N}_{e}\right)_{j k}\left(\mathbf{N}_{e, m_{1} n_{2} q_{l}}\right)_{j a}\right] \mathrm{d} V, \\
& \left(\mathbf{H}_{12}\right)_{k a, q_{l}}=2 \rho \int_{V_{0}}\left[\left(\mathbf{N}_{e, q_{l}}\right)_{j k}\left(\mathbf{N}_{e, n_{1} m_{2}}\right)_{j a}+\left(\mathbf{N}_{e}\right)_{j k}\left(\mathbf{N}_{e, n_{1} m_{2} q_{l}}\right)_{j a}\right] \mathrm{d} V, \\
& \left(\mathbf{H}_{13}\right)_{k a, q_{l}}=2 \rho \int_{V_{0}}\left[\left(\mathbf{N}_{e, q_{l}}\right)_{j k}\left(\mathbf{N}_{e, n_{1} n_{2}}\right)_{j a}+\left(\mathbf{N}_{e}\right)_{j k}\left(\mathbf{N}_{e, n_{1} n_{2} q_{l}}\right)_{j a}\right] \mathrm{d} V, \\
& \left(\mathbf{H}_{14}\right)_{k a, q_{l}}=2 \rho \int_{V_{0}}\left[\left(\mathbf{N}_{e, q_{l}}\right)_{j k}\left(\mathbf{N}_{e, m_{2} n_{2}}\right)_{j a}+\left(\mathbf{N}_{e}\right)_{j k}\left(\mathbf{N}_{e, m_{2} n_{2} q_{l}}\right)_{j a}\right] \mathrm{d} V,
\end{aligned}
$$

and

$$
\begin{aligned}
& \left(\mathbf{H}_{15}\right)_{k b a, q_{l}}=2 \rho \int_{V_{0}}\left[\left(\mathbf{N}_{e, q_{k} q_{l}}\right)_{j b}\left(\mathbf{N}_{e, m_{1}}\right)_{j a}+\left(\mathbf{N}_{e, q_{k}}\right)_{j b}\left(\mathbf{N}_{e, m_{1} q_{l}}\right)_{j a}\right] \mathrm{d} V, \\
& \left(\mathbf{H}_{16}\right)_{k b a, q_{l}}=2 \rho \int_{V_{0}}\left[\left(\mathbf{N}_{e, q_{k} q_{l}}\right)_{j b}\left(\mathbf{N}_{e, n_{1}}\right)_{j a}+\left(\mathbf{N}_{e, q_{k}}\right)_{j b}\left(\mathbf{N}_{e, n_{1} q_{l}}\right)_{j a}\right] \mathrm{d} V, \\
& \left(\mathbf{H}_{17}\right)_{k b a, q_{l}}=2 \rho \int_{V_{0}}\left[\left(\mathbf{N}_{e, q_{k} q_{l}}\right)_{j b}\left(\mathbf{N}_{e, m_{2}}\right)_{j a}+\left(\mathbf{N}_{e, q_{k}}\right)_{j b}\left(\mathbf{N}_{e, m_{2} q_{l}}\right)_{j a}\right] \mathrm{d} V, \\
& \left(\mathbf{H}_{18}\right)_{k b a, q_{l}}=2 \rho \int_{V_{0}}\left[\left(\mathbf{N}_{e, q_{k} q_{l}}\right)_{j b}\left(\mathbf{N}_{e, n_{2}}\right)_{j a}+\left(\mathbf{N}_{e, q_{k}}\right)_{j b}\left(\mathbf{N}_{e, n_{2} q_{l}}\right)_{j a}\right] \mathrm{d} V, \\
& \left(\mathbf{H}_{19}\right)_{k b a, q_{l}}=\rho \int_{V_{0}}\left[\left(\mathbf{N}_{e, q_{k} q_{l}}\right)_{j b}\left(\mathbf{N}_{e, m_{1} m_{1}}\right)_{j a}+\left(\mathbf{N}_{e, q_{k}}\right)_{j b}\left(\mathbf{N}_{e, m_{1} m_{1} q_{l}}\right)_{j a}\right] \mathrm{d} V, \\
& \left(\mathbf{H}_{20}\right)_{k b a, q_{l}}=\rho \int_{V_{0}}\left[\left(\mathbf{N}_{e, q_{k} q_{l}}\right)_{j b}\left(\mathbf{N}_{e, n_{1} n_{1}}\right)_{j a}+\left(\mathbf{N}_{e, q_{k}}\right)_{j b}\left(\mathbf{N}_{e, n_{1} n_{1} q_{l}}\right)_{j a}\right] \mathrm{d} V, \\
& \left(\mathbf{H}_{21}\right)_{k b a, q_{l}}=\rho \int_{V_{0}}\left[\left(\mathbf{N}_{e, q_{k} q_{l}}\right)_{j b}\left(\mathbf{N}_{e, m_{2} m_{2}}\right)_{j a}+\left(\mathbf{N}_{e, q_{k}}\right)_{j b}\left(\mathbf{N}_{e, m_{2} m_{2} q_{l}}\right)_{j a}\right] \mathrm{d} V, \\
& \left(\mathbf{H}_{22}\right)_{k b a, q_{l}}=\rho \int_{V_{0}}\left[\left(\mathbf{N}_{e, q_{k} q_{l}}\right)_{j b}\left(\mathbf{N}_{e, n_{2} n_{2}}\right)_{j a}+\left(\mathbf{N}_{e, q_{k}}\right)_{j b}\left(\mathbf{N}_{e, n_{2} n_{2} q_{l}}\right)_{j a}\right] \mathrm{d} V, \\
& \left(\mathbf{H}_{23}\right)_{k b a, q_{l}}=2 \rho \int_{V_{0}}\left[\left(\mathbf{N}_{e, q_{k} q_{l}}\right)_{j b}\left(\mathbf{N}_{e, m_{1} n_{1}}\right)_{j a}+\left(\mathbf{N}_{e, q_{k}}\right)_{j b}\left(\mathbf{N}_{e, m_{1} n_{1} q_{l}}\right)_{j a}\right] \mathrm{d} V, \\
& \left(\mathbf{H}_{24}\right)_{k b a, q_{l}}=2 \rho \int_{V_{0}}\left[\left(\mathbf{N}_{e, q_{k} q_{l}}\right)_{j b}\left(\mathbf{N}_{e, m_{1} m_{2}}\right)_{j a}+\left(\mathbf{N}_{e, q_{k}}\right)_{j b}\left(\mathbf{N}_{e, m_{1} m_{2} q_{l}}\right)_{j a}\right] \mathrm{d} V, \\
& \left(\mathbf{H}_{25}\right)_{k b a, q_{l}}=2 \rho \int_{V_{0}}\left[\left(\mathbf{N}_{e, q_{k} q_{l}}\right)_{j b}\left(\mathbf{N}_{e, m_{1} n_{2}}\right)_{j a}+\left(\mathbf{N}_{e, q_{k}}\right)_{j b}\left(\mathbf{N}_{e, m_{1} n_{2} q_{l}}\right)_{j a}\right] \mathrm{d} V, \\
& \left(\mathbf{H}_{26}\right)_{k b a, q_{l}}=2 \rho \int_{V_{0}}\left[\left(\mathbf{N}_{e, q_{k} q_{l}}\right)_{j b}\left(\mathbf{N}_{e, n_{1} m_{2}}\right)_{j a}+\left(\mathbf{N}_{e, q_{k}}\right)_{j b}\left(\mathbf{N}_{e, n_{1} m_{2} q_{l}}\right)_{j a}\right] \mathrm{d} V, \\
& \left(\mathbf{H}_{27}\right)_{k b a, q_{l}}=2 \rho \int_{V_{0}}\left[\left(\mathbf{N}_{e, q_{k} q_{l}}\right)_{j b}\left(\mathbf{N}_{e, n_{1} n_{2}}\right)_{j a}+\left(\mathbf{N}_{e, q_{k}}\right)_{j b}\left(\mathbf{N}_{e, n_{1} n_{2} q_{l}}\right)_{j a}\right] \mathrm{d} V, \\
& \left(\mathbf{H}_{28}\right)_{k b a, q_{l}}=2 \rho \int_{V_{0}}\left[\left(\mathbf{N}_{e, q_{k} q_{l}}\right)_{j b}\left(\mathbf{N}_{e, m_{2} n_{2}}\right)_{j a}+\left(\mathbf{N}_{e, q_{k}}\right)_{j b}\left(\mathbf{N}_{e, m_{2} n_{2} q_{l}}\right)_{j a}\right] \mathrm{d} V .
\end{aligned}
$$


In Eqs. (A.6) and (A.7), the subscripts $a, b=1,2, \ldots, 36$ and $j=1,2,3$.

The matrices in Eqs. (A.2)-(A.7) are all invariant and can be computed and stored with sparse matrix technique in the preprocessing procedure to greatly improve the computation efficiency.

\section{References}

1 Yang, C.J., Hong, D.F., Ren, G.X., Zhao, Z.H.: Cable installation simulation by using a multibody dynamic model. Multibody System Dynamics. 30(4), 433-447 (2013)

2 Hong, D.F., Tang, J.L., Ren, G.X.: Dynamic modeling of mass-flowing linear medium with large amplitude displacement and rotation. Journal of Fluids and Structures. 27(8), 1137-1148 (2011)

3 Tang, J.L., Ren, G.X., Zhu, W.D., Ren, H.: Dynamics of variable-length tethers with application to tethered satellite deployment. Communications in Nonlinear Science and Numerical Simulation. 16(8), 3411-3424 (2011)

4 Escalona, J.L.: An arbitrary Lagrangian-Eulerian discretization method for modeling and simulation of reeving systems in multibody dynamics. Mechanism and Machine Theory. 112 1-21 (2017)

5 Du, J.L., Cui, C.Z., Bao, H., Qiu, Y.Y.: Dynamic analysis of cable-driven parallel manipulators using a variable length finite element. Journal of Computational and Nonlinear Dynamics. 10(1), 011013 (2015)

6 Sakamoto, H., Miyazaki, Y., Mori, O.: Transient dynamic analysis of gossamer appendage deployment using nonlinear finite element method. Journal of Spacecraft and Rockets. 48(5), 881-890 (2011)

7 Zhao, J., Tian, Q., Hu, H.Y.: Deployment dynamics of a simplified spinning IKAROS solar sail via absolute coordinate based method. Acta Mechanica Sinica. 29(1), 132-142 (2013)

8 https://www.orbitalatk.com/space-systems/space-components/deployables/default.aspx

9 Wang, J., Qi, Z.H., Wang, G.: Hybrid modeling for dynamic analysis of cable-pulley systems with time-varying length cable and its application. Journal of Sound and Vibration. 406 277-294 (2017)

10 Longva, V., Sævik, S.: A Lagrangian-Eulerian formulation for reeling analysis of historydependent multilayered beams. Computers \& Structures. 146 44-58 (2015)

11 Pechstein, A., Gerstmayr, J.: A Lagrange-Eulerian formulation of an axially moving beam based on the absolute nodal coordinate formulation. Multibody System Dynamics. 30(3), 343-358 (2013)

12 Gross, D., Messner, D.: The able deployable articulated mast-enabling technology for the shuttle radar topography mission. 33rd Aerospace Mechanisms Symposium, Pasadena, California, May 19-21 (1999)

13 Bendsøe, M.P., Kikuchi, N.: Generating optimal topologies in structural design using a homogenization method. Computer Methods in Applied Mechanics and Engineering. 71(2), 197-224 (1988)

14 Sigmund, O., Maute, K.: Topology optimization approaches: a comparative review. 
Structural and Multidisciplinary Optimization. 48(6), 1031-1055 (2013)

15 Sun, J.L., Tian, Q., Hu, H.Y.: Topology optimization based on level set for a flexible multibody system modeled via ANCF. Structural and Multidisciplinary Optimization. 55 1159-1177 (2017)

16 Tromme, E., Tortorelli, D., Brüls, O., Duysinx, P.: Structural optimization of multibody system components described using level set techniques. Structural and Multidisciplinary Optimization. 52(5), 959-971 (2015)

17 Held, A., Nowakowski, C., Moghadasi, A., Seifried, R., Eberhard, P.: On the influence of model reduction techniques in topology optimization of flexible multibody systems using the floating frame of reference approach. Structural and Multidisciplinary Optimization. 53(1), 67-80 (2016)

18 Moghadasi, A., Held, A., Seifried, R.: Modeling of revolute joints in topology optimization of flexible multibody systems. Journal of Computational and Nonlinear Dynamics. 12(1), 011015 (2017)

19 Sun, J.L., Tian, Q., Hu, H.Y.: Topology optimization of a three-dimensional flexible multibody system via moving morphable components. Journal of Computational and Nonlinear Dynamics. 13(2), 021010 (2018)

20 Shabana, A.A.: An absolute nodal coordinates formulation for the large rotation and deformation analysis of flexible bodies. Report. No. MBS96-1-UIC, University of Illinois at Chicago (1996)

21 Simo, J.C., Vu-Quoc, L.: On the dynamics of flexible beams under large overall motions: the plane case. Part I and II. Journal of Applied Mechanics. 53(4), 849-863 (1986)

22 Ding, J.Y., Wallin, M., Wei, C., Recuero, A.M., Shabana, A.A.: Use of independent rotation field in the large displacement analysis of beams. Nonlinear Dynamics. 76(3), 1829-1843 (2014)

23 Hayashi, H., Takehara, S., Terumichi, Y.: Numerical approach for flexible body motion with large displacement and time-varying length. The 3rd Joint International Conference on Multibody System Dynamics and the 7th Asian Conference on Multibody Dynamics, BEXCO, Busan, Korea, June 30-July 3 (2014)

24 Terumichi, Y., Kaczmarczyk, S., Sogabe, K.: Numerical approach in the analysis of flexible body motion with time-varying length and large displacement using multiple time scales. The 1st Joint International Conference on Multibody System Dynamics, Lappeenranta, Finland, May 25-27 (2010)

25 Hong, D.F., Ren, G.X.: A modeling of sliding joint on one-dimensional flexible medium. Multibody System Dynamics. 26(1), 91-106 (2011)

26 Hyldahl, P., Mikkola, A., Balling, O.: A thin plate element based on the combined arbitrary Lagrange-Euler and absolute nodal coordinate formulations. Proceedings of the Institution of Mechanical Engineers, Part K: Journal of Multi-Body Dynamics. 227(3), 211-219 (2013)

27 Sun, J.L., Tian, Q., Hu, H.Y.: Structural optimization of flexible components in a flexible multibody system modeled via ANCF. Mechanism and Machine Theory. 104 59-80 (2016)

28 Kang, B.S., Park, G.J., Arora, J.S.: Optimization of flexible multibody dynamic systems using the equivalent static load method. AIAA Journal. 43(4), 846-852 (2005)

29 Hong, E.P., You, B.J., Kim, C.H., Park, G.J.: Optimization of flexible components of 
multibody systems via equivalent static loads. Structural and Multidisciplinary Optimization. 40(1-6), 549-562 (2010)

30 Tromme, E., Sonneville, V., Brüls, O., Duysinx, P.: On the equivalent static load method for flexible multibody systems described with a nonlinear finite element formalism. International Journal for Numerical Methods in Engineering. 108(6), 646-664 (2016)

31 Tromme, E., Sonneville, V., Guest, J.K., Brüls, O.: System-wise equivalent static loads for the design of flexible mechanisms. Computer Methods in Applied Mechanics and Engineering. 329 312-331 (2018)

32 Tromme, E., Brüls, O., Duysinx, P.: Weakly and fully coupled methods for structural optimization of flexible mechanisms. Multibody System Dynamics. 38(4), 391-417 (2016)

33 Zhang, W.S., Li, D., Yuan, J., Song, J.F., Guo, X.: A new three-dimensional topology optimization method based on moving morphable components (MMCs). Computational Mechanics. 69(4), 647-665 (2017)

34 Zhang, W.S., Yuan, J., Zhang, J., Guo, X.: A new topology optimization approach based on moving morphable components (MMC) and the ersatz material model. Structural and Multidisciplinary Optimization. 53(6), 1243-1260 (2016)

35 Guo, X., Zhang, W.S., Zhang, J., Yuan, J.: Explicit structural topology optimization based on moving morphable components (MMC) with curved skeletons. Computer Methods in Applied Mechanics and Engineering. 310 711-748 (2016)

36 Zhang, W.S., Li, D., Zhang, J., Guo, X.: Minimum length scale control in structural topology optimization based on the moving morphable components (MMC) approach. Computer Methods in Applied Mechanics and Engineering. 311 327-355 (2016)

37 Guo, X., Zhang, W.S., Zhong, W.L.: Doing topology optimization explicitly and geometrically-a new moving morphable components based framework. Journal of Applied Mechanics. 81(8), 081009 (2014)

38 Zhao, J., Tian, Q., Hu, H.Y.: Modal analysis of a rotating thin plate via absolute nodal coordinate formulation. Journal of Computational and Nonlinear Dynamics. 6(4), 041013 (2011)

39 Dufva, K., Shabana, A.A.: Analysis of thin plate structures using the absolute nodal coordinate formulation. Proceedings of the Institution of Mechanical Engineers, Part K: Journal of Multi-Body Dynamics. 219(4), 345-355 (2005)

40 Sanborn, G.G., Choi, J., Choi, J.H.: Curve-induced distortion of polynomial space curves, flat-mapped extension modeling, and their impact on ANCF thin-plate finite elements. Multibody System Dynamics. 26(2), 191-211 (2011)

41 Garcia-Vallejo, D., Mayo, J., Escalona, J.L.: Efficient evaluation of the elastic forces and the jacobian in the absolute nodal coordinate formulation. Nonlinear Dynamics. 35(4), 313-329 (2004)

42 Arnold, M., Brüls, O.: Convergence of the generalized- $\alpha$ scheme for constrained mechanical systems. Multibody System Dynamics. 18 185-202 (2007)

43 Tian, Q., Zhang, Y.Q., Chen, L.P., Yang, J.Z.: Simulation of planar flexible multibody systems with clearance and lubricated revolute joints. Nonlinear Dynamics. 60(4), 489$511(2010)$

44 Tian, Q., Lou, J., Mikkola, A.: A new elastohydrodynamic lubricated spherical joint model for rigid-flexible multibody dynamics. Mechanism and Machine Theory. 107 210- 
$228(2017)$

45 Tian, Q., Sun, Y.L., Liu, C., Hu, H.Y., Flores, P.: Elastohydrodynamic lubricated cylindrical joints for rigid-flexible multibody dynamics. Computers \& Structures. 114115 106-120 (2013)

46 Yang, Z.J., Chen, X., Kelly, R.: A topological optimization approach for structural design of a high-speed low-load mechanism using the equivalent static loads method. International Journal for Numerical Methods in Engineering. 89(5), 584-598 (2012)

47 Luo, Z., Yang, J.Z., Chen, L.P., Zhang, Y.Q., Abdel-Malek, K.: A new hybrid fuzzy-goal programming scheme for multi-objective topological optimization of static and dynamic structures under multiple loading conditions. Structural and Multidisciplinary Optimization. 31(1), 26-39 (2006)

48 Svanberg, K.: The method of moving asymptotes - a new method for structural optimization. International Journal for Numerical Methods in Engineering. 24(2), 359373 (1987)

49 Dmitrochenko, O.N., Pogorelov, D.Y.: Generalization of plate finite elements for absolute nodal coordinate formulation. Multibody System Dynamics. 10 17-43 (2003)

50 Luo, K., Liu, C., Tian, Q., Hu, H.: Nonlinear static and dynamic analysis of hyper-elastic thin shells via the absolute nodal coordinate formulation. Nonlinear Dynamics. 85(2), 949-971 (2016)

51 Dmitrochenko, O., Mikkola, A.: Two simple triangular plate elements based on the absolute nodal coordinate formulation. Journal of Computational and Nonlinear Dynamics. 3(4), 041012 (2008)

52 Olshevskiy, A., Dmitrochenko, O., Dai, M.D., Kim, C.: The simplest 3-, 6- and 8-noded fully-parameterized ANCF plate elements using only transverse slopes. Multibody System Dynamics. 34(1), 23-51 (2015)

53 Olshevskiy, A., Dmitrochenko, O., Lee, S., Kim, C.: A triangular plate element 2343 using second-order absolute-nodal-coordinate slopes: numerical computation of shape functions. Nonlinear Dynamics. 74(3), 769-781 (2013)

54 García-Vallejo, D., Mikkola, A.M., Escalona, J.L.: A new locking-free shear deformable finite element based on absolute nodal coordinates. Nonlinear Dynamics. 50(1-2), 249264 (2007)

55 Nachbagauer, K., Pechstein, A.S., Irschik, H., Gerstmayr, J.: A new locking-free formulation for planar, shear deformable, linear and quadratic beam finite elements based on the absolute nodal coordinate formulation. Multibody System Dynamics. 26(3), 245$263(2011)$

56 De Jalón, J.G., Bayo, E.: Kinematic and dynamic simulation of multibody systems the real-time challenge, Springer, New York (1994) 